\section{SPAL 30.2 $(2021)$}

\section{$103-123$}

ISSN: $1133-4525$

ISSN-e: 2255-3924

\section{FECHA RECEPCIÓN}

05/03/2020

FECHA ACEPTACIÓN

$19 / 04 / 2021$

\section{El proceso de fabricación de los cuencos con borde biselado mesopotámicos. Nuevas aportaciones desde la arqueología experimental}

\author{
THE MANUFACTURING PROCESS OF MESOPOTAMIAN BEVELLED RIM BOWLS. \\ NEW CONTRIBUTIONS FROM EXPERIMENTAL ARCHAEOLOGY
}

\section{Juan Luis Montero Fenollós}

Departamento de Humanidades, Universidade da Coruña fenollos@udc.es (1) 0000-0002-0689-3765 P K-6218-2014 (Responsable de correspondencia)

\section{Jorge Sanjurjo Sánchez}

Instituto Universitario de Xeoloxía, Universidade da Coruña jorge.sanjurjo.sanchez@udc.es (1) 0000-0002-7559-8647 P E-4404-2011

Resumen Los cuencos con borde biselado constituyen un signo de identidad de la cultura de Uruk en Mesopotamia y de su expansión territorial en otras regiones limítrofes de Oriente Próximo, durante la segunda mitad del IV milenio a.C. Sin embargo, en la actualidad conocemos mal tanto la función de este tipo de vasos de cerámica como el método con el que fueron elaborados por los artesanos de la época. A partir del análisis morfológico, geoquímico y de la temperatura de cocción, así como de la observación por Tomografía Axial Computarizada (TAC) y de la ayuda de la arqueología experimental, se hace una nueva propuesta sobre el proceso de elaboración de los cuencos con borde biselados urukeos, fundamentada en el uso de un molde portátil.

Palabras clave Mesopotamia, Calcolítico Reciente, cerámica, molde, pan

Abstract Bevelled rim bowls (BRB) are a sign of identity of the Uruk culture during the second half of the 4th millennium BC in Mesopotamia and of its territorial expansion in other bordering regions of the Near East. However, today we have a poor understanding of both the function of this type of ceramic vessel and the method of production used by the craftsmen of that period. On the basis of morphological and geochemical analysis, the firing temperature, CT observations (Computed Tomography) and the help of experimental archaeology, a new proposal is made for the production process of the bevelled rim bowls with the help of a portable mould.

Key words Mesopotamia, Late Chalcolithic period, pottery, mould, bread.

Montero Fenollós, J. L. y Sanjurjo Sánchez, J. (2021): “El proceso de fabricación de los cuencos con borde biselado mesopotámicos. Nuevas aportaciones desde la arqueología experimental", Spal, 30.2, pp. 103-123.

https://dx.doi.org/10.12795/spal.2021.i30.19 
SPAL 30.2

(2021)

\section{$103-123$}

ISSN: $1133-4525$

ISSN-e: 2255-3924

\section{INTRODUCCIÓN}

La cultura de Uruk, en particular el período Uruk Medio y Reciente en Mesopotamia, también conocido como Calcolítico Reciente 4-5 en el contexto general del Oriente Próximo antiguo (Rothman, 2001, p. 9), se conformó como una etapa determinante en los albores de la civilización urbana (Liverani, 1998; Algaze, 2008; Crüsemann et al., 2019). Esta cultura, originaria de la Mesopotamia meridional, conoció entre 3600 y 3000 a.C. un proceso de expansión geográfica tanto hacia el norte (Siria y Anatolia) como hacia el este (Irán). La principal consecuencia de esta difusión fue la aparición de una vasta base territorial unificada bajo el mismo horizonte cultural, aunque no exenta de discontinuidades y de diferencias, sobre todo en aquellos territorios donde coexistió con culturas calcolíticas locales con alto nivel de desarrollo (como en Arslantepe).

La presencia a centenares de kilómetros de la ciudad de Uruk, en el sur del actual Iraq, de enclaves que han sido definidos como "auténticos clones culturales" (Butterlin, 2018, p. 409) de la metrópoli bajo-mesopotámica ha hecho pensar en la existencia e implantación de un sistema de verdaderas colonias urukeas. Más allá de este sistema de implantación colonial, existía un vasto espacio con asentamientos en los que han aparecido mezclados productos locales y objetos propios a la cultura de Uruk. Estos hallazgos arqueológicos han alimentado un debate entre los miembros de la comunidad científica sobre el modelo seguido por la cultura de Uruk en su proceso de expansión. Los términos de la controversia se han centrado en conceptos como: sistema-mundo, colonización, aculturación y globalización (Algaze, 2004).

Las verdaderas razones que dieron lugar a la expansión territorial urukea continúan siendo una cuestión controvertida para la que se han propuesto diversas explicaciones (Butterlin, 2018, pp. 407-427). El peso de la discusión ha recaído sobre la identificación de lo que fue y de lo que estuvo ligado a la cultura de Uruk. De esta manera, los investigadores han intentado establecer cuál fue el conjunto cultural genuinamente urukeo. Entre los componentes fundamentales del mismo se hallaban la cerámica, el urbanismo, la escritura pictográfica, los sellos cilindricos y la arquitectura monumental. De acuerdo, con estos principios, se ha pensado que solo los lugares de hábitat que contenían la totalidad de este "kit colonial" podían ser considerados verdaderamente urukeos y, por tanto, colonias auténticas. Por el contrario, los enclaves donde la presencia de este conjunto era parcial y se documenta la convivencia con elementos locales, serían el resultado de un proceso más o menos complejo de aculturación o de influencias externas.

Entre la cultura material genuina del período Uruk Medio y Reciente se encuentra un tipo de cerámica, denominado cuenco con borde biselado, que representa entre el 50 y el $90 \%$ de toda la cerámica hallada en los yacimientos de esta fase arqueológica, según los autores consultados (Chazan y Lehner, 1990, p. 26; Hopkinson 2007, p. 47; Butterlin, 2018, p. 409). En lo que no hay controversia es en el hecho de considerar este tipo de vasos como el mejor indicativo material, o fósil guía, del estilo definido como urukeo auténtico. Tal es así, que su forma y su aspecto singular se han convertido en el emblema identitario de toda una cultura, que floreció en la antigua Mesopotamia en la segunda mitad del IV milenio a.C. Este vaso es conocido como écuelle grossière en francés, Glockentopf en alemán y bevelled rim bowl en inglés. Esta última denominación (abreviada en BRB) es la que se ha impuesto en el campo de los estudios de arqueología del Próximo Oriente. 
SPAL 30.2 $(2021)$

\section{3-123}

ISSN: $1133-4525$ ISSN-e: 2255-3924
A pesar de ser un objeto muy común en el registro arqueológico de los asentamientos ligados a la expansión territorial de Uruk (fig. 1), son rios de tinta los que han corrido a la hora de dilucidar la función de este tipo de cuencos (en adelante BRB). A día de hoy, no hay unanimidad entre los investigadores cuando se trata de establecer cuál fue el uso para el que fueron diseñados y fabricados estos cuencos. Por esta razón, nos tenemos que mover en el campo de las hipótesis. Dada la multiplicidad de contextos en los que fueron hallados, se han presentado como testigos materiales de: la vida religiosa (ofrendas en templos y tumbas), de la vida cotidiana (preparación de diversos tipos de alimentos) o de la organización socioeconómica (raciones para el pago de impuestos y salarios) de la época (Le Brun, 1980, pp. 62-66; Faivre, 2009, p.308; Porter, 2012, pp. 95-96). Entre las interpretaciones más recientes, se hallan aquellas que proponen su uso como moldes para elaborar pan con levadura (Goulder, 2010; Montero y Sanjurjo, 2016, pp. 299-302) o como vasos para distribuir cerveza o un derivado de esta (una especie de gachas de cebada) entre trabajadores de las instituciones urukeas (Bernbeck, 2012, p. 54; Pollock, 2012, p. 160-161). Aunque el debate sobre la función de los BRB continúa abierto, en la actualidad, los últimos estudios analíticos en laboratorio permiten excluir algunas de las hipótesis propuestas y parecen avalar la tesis del pan con levadura (Sanjurjo et al., 2018a). En este sentido, es importante resaltar que el pictograma GAR, usado en los textos de Uruk, es un cuenco que formalmente recuerda el aspecto de los BRB. Este signo (leído ninda en sumerio) ha sido traducido por hogaza de pan (Márquez, 2009, pp. 144-146).

Con el objetivo de arrojar luz sobre la cultura de Uruk y su expansión geográfica por el valle del Medio Éufrates sirio, la Universidade da Coruña y la Dirección General de Antigüedades y Museos de Damasco pusieron en marcha la excavación arqueológica de Tell Humeida, en la entrada de la garganta basáltica de Khanuqa (fig. 2a y b). Se trata de un yacimiento situado en la ribera oriental del Éufrates, $75 \mathrm{~km}$ al norte de Deir ez-Zor,

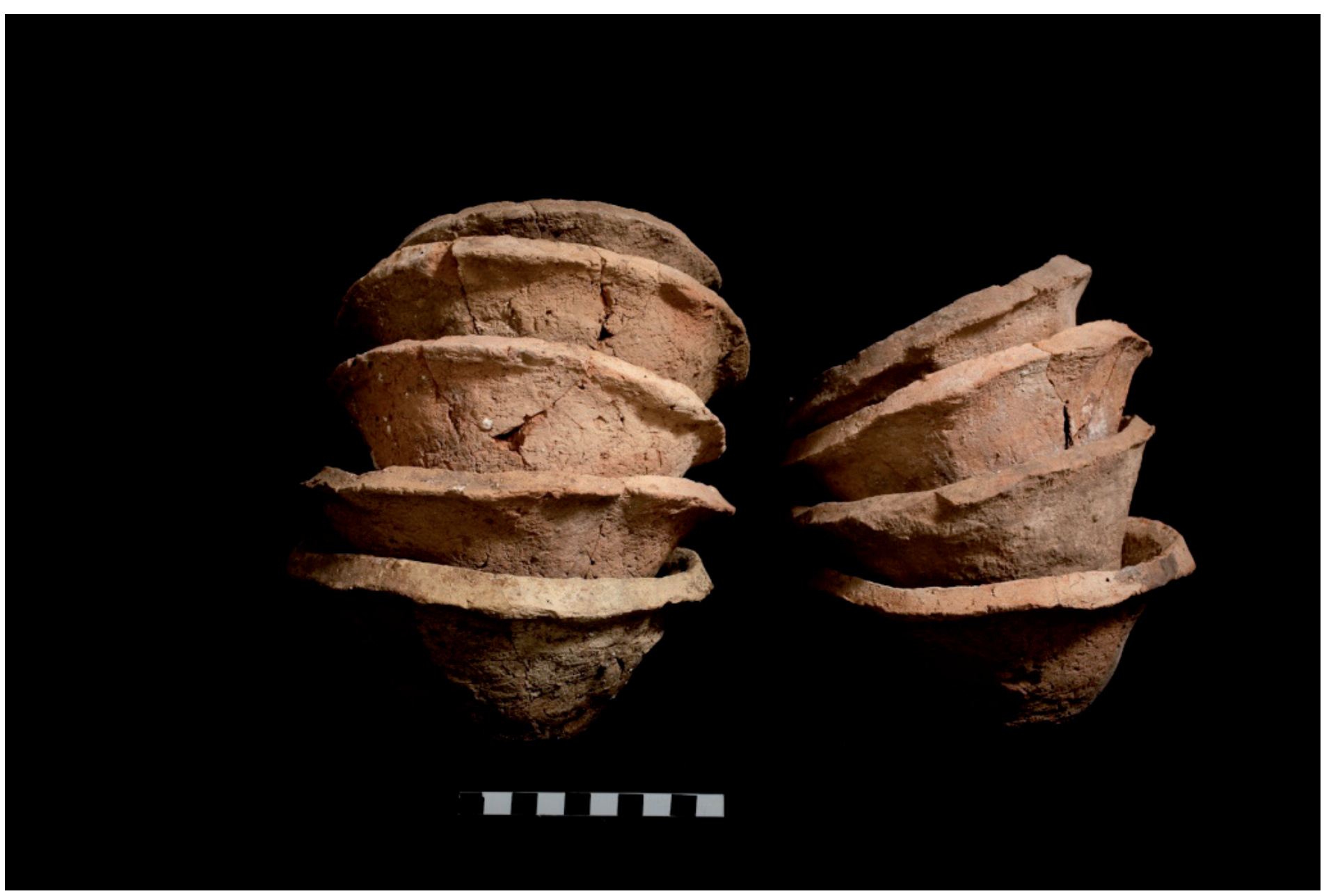

Figura 1. Ejemplares de BRB procedentes de Tell Humeida, Siria (Foto: Eloy Taboada-PAMES). 
SPAL 30.2 (2021)

\section{3-123}

ISSN: 1133-4525

ISSN-e: 2255-3924

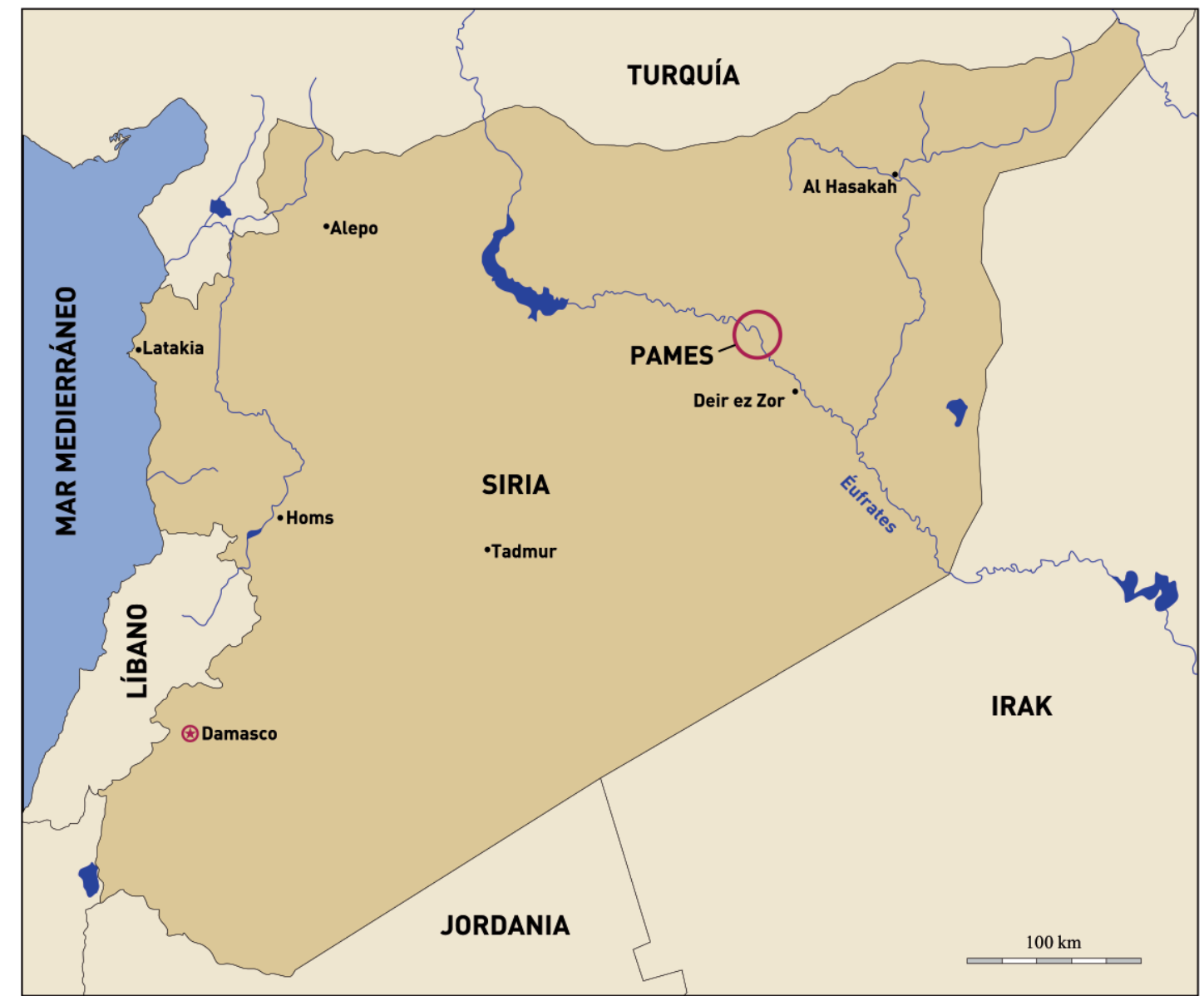

Figura 2a y 2b. Mapa de situación del Proyecto Arqueológico Medio Éufrates Sirio (Mapa: Francisco Bescós-PAMES. Cartografía base: Dirección General de Antigüedades y Museos de Siria); Ubicación de Tell Humeida en la garganta de Khanuqa, en el Medio Éufrates (Mapa: José Miguel Gaspar-PAMES. Base cartográfica: Satélite Landsat 7, US Geological Survey)..

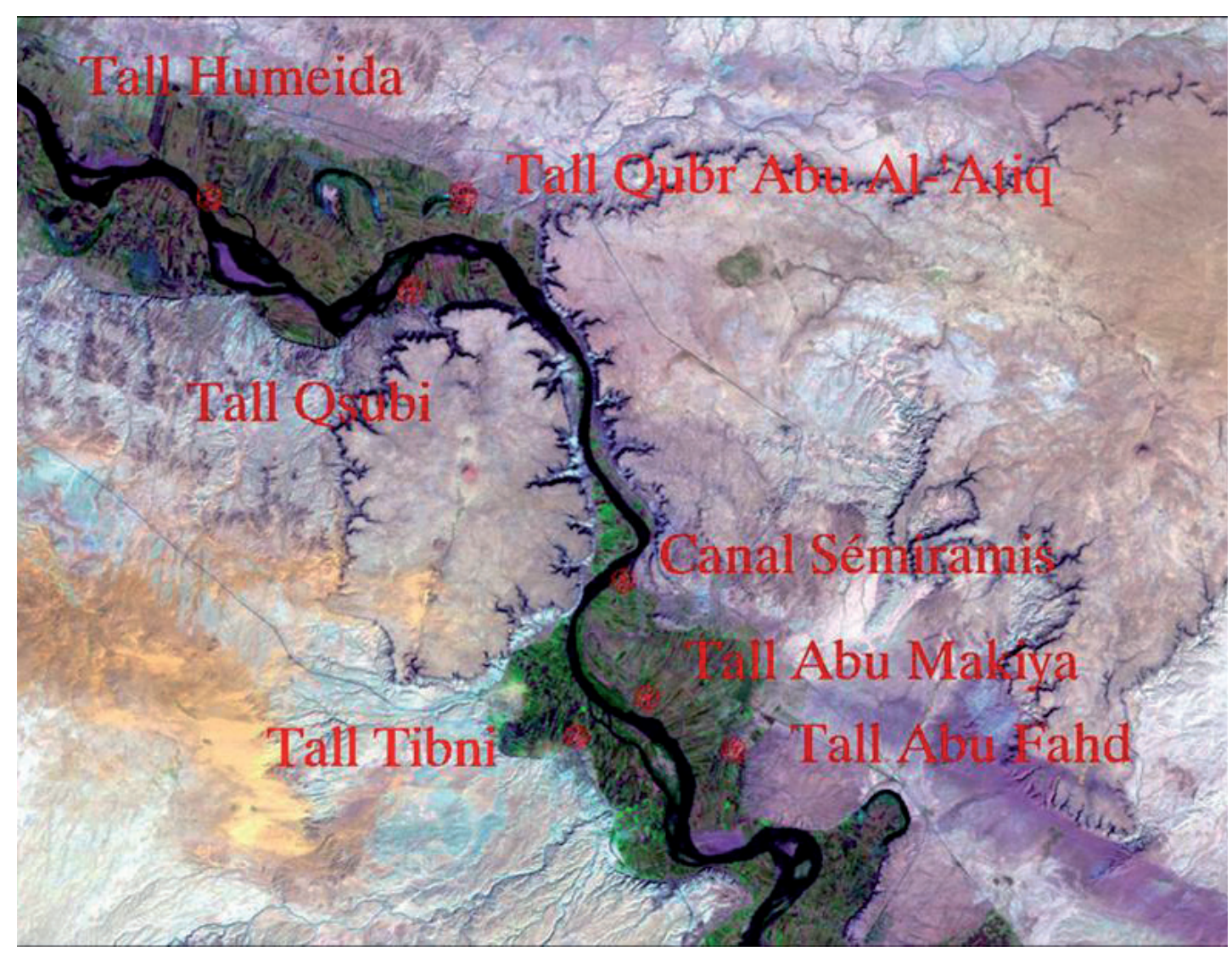

que ocupa una superficie aproximada de 6 ha distribuidas en dos partes bien diferenciadas: una colina principal de forma casi circular (de $150 \mathrm{~m}$ de diámetro) y una zona baja que se extiende hacia el este.

Tras varias campañas de prospección, en 2011, se realizó un sondeo estratigráfico en la ladera occidental de la colina principal de este asentamiento, una actuación que sirvió para certificar la existencia de dos grandes períodos de ocupación: el primero paleobizantino y el segundo Uruk Medio. A este último período pertenece una unidad estratigráfica (UE-1006) caracterizada por la abundante presencia de carbón vegetal, de huesos de animal y de cerámica, entre ella numerosos BRB (fig. 3). Por su naturaleza, se trata de un basurero, con una potencia superior a los $2 \mathrm{~m}$ (Montero, 2011). 
SPAL 30.2 (2021)

\section{$103-123$}

ISSN: $1133-4525$ ISSN-e: 2255-3924

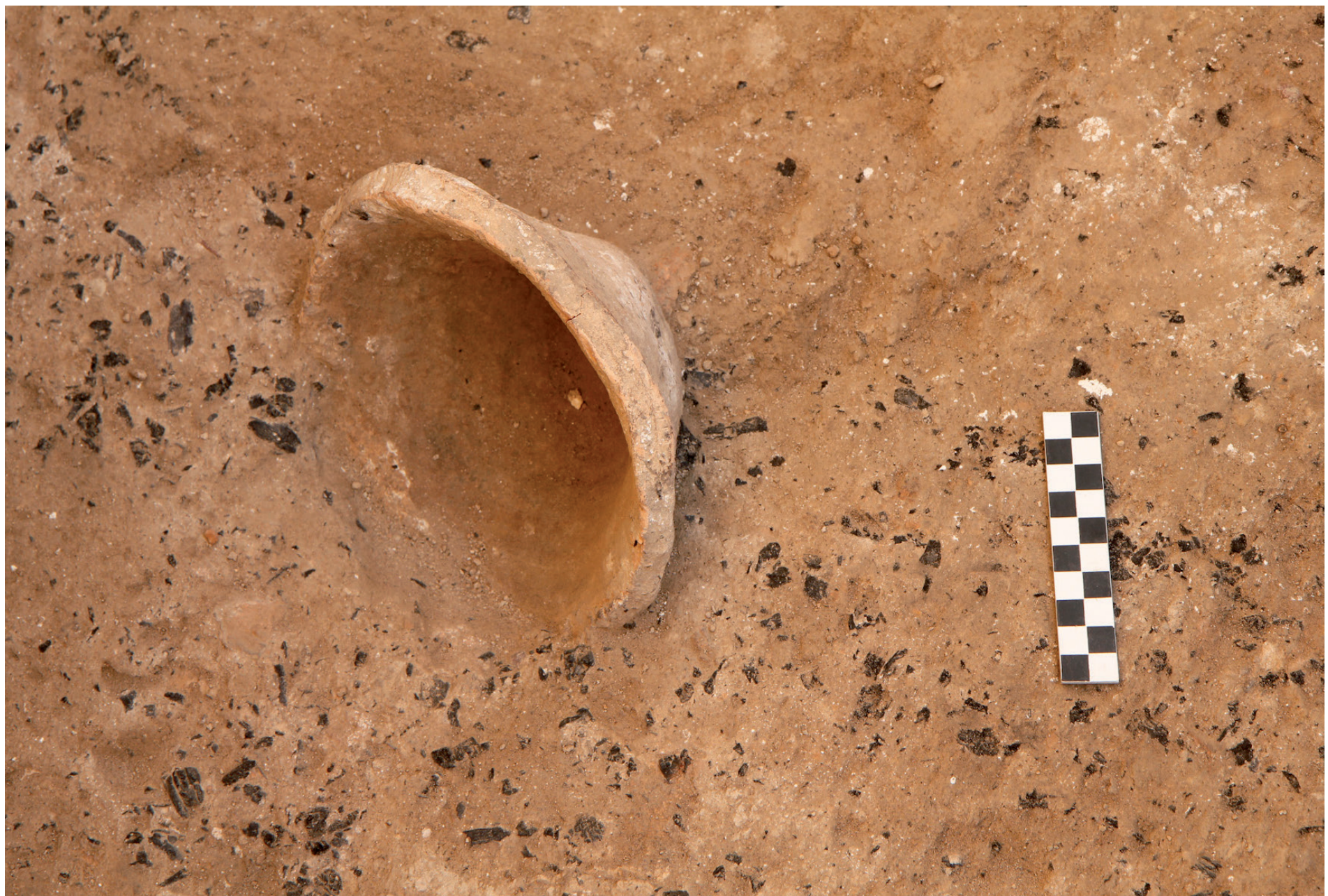

Figura 3. Detalle de un BRB completo en el basurero de Tell Humeida (Foto: Eloy Taboada-PAMES).

Las dataciones radiocarbónicas realizadas a dos muestras de carbones por medio de espectrometría de masas acoplada a acelerador de partículas (AMS), en la Universidad de Uppsala, indicaron edades no calibradas de 4917 440 yr BP (Ua-42144) y de $4811 \pm 34$ BP (Ua-42140), que corresponden respectivamente a los intervalos 3780-3640 BCE cal y 3660-3520 BCE cal.

Aprovechando la presencia masiva de BRB en el vertedero urukeo de Tell Humeida, y teniendo en cuenta la problemática que rodea a este objeto, se decidió organizar un programa de investigación con el que aportar nuevos datos sobre la expansión de la cultura de Uruk a lo largo del valle del Medio Éufrates sirio, en la segunda mitad del IV milenio a.C. La investigación se ha centrado en el estudio de tres aspectos relacionados con los BRB: la composición geoquímica de la materia prima utilizada, el proceso y técnica de fabricación, y la función para que fueron elaborados. Además de la documentación procedente del Tell Humeida, cuya excavación se vio paralizada por la guerra que asola actualmente Siria, se solicitaron muestras de BRB a otros proyectos arqueológicos que habían trabajado en Siria e Iraq y se estudiaron las colecciones de BRB conservadas en el Musée du Louvre y los Musées Royaux d’Art et d'Histoire de Bruselas.

\section{EL PROCESO DE PRODUCCIÓN DE LOS BRB URUKEOS}

\subsection{Hipótesis explicativas}

Sobre la fabricación de los BRB urukeos se han propuesto hasta la fecha dos técnicas distintas: o bien a mano, o bien con la ayuda de un molde, que podía estar excavado en el suelo o ser portátil (de cerámica, yeso u otro material de tipo perecedero). 
SPAL 30.2

(2021)

\section{$103-123$}

ISSN: $1133-4525$ ISSN-e: 2255-3924
Apoyándose en el estudio de los BRB hallados en Yebel Aruda (norte de Siria) y en una serie de experimentos hechos en el Institute of Pottery Technology de Leiden, se ha planteado que estos vasos fueron elaborados simplemente a mano (Kalsbeek, 1980, pp. 1-5) partiendo de un cono de arcilla. Con la única ayuda de sus manos, el artesano abría una cavidad en el mismo, que se iba agrandando progresivamente a partir de un movimiento circular. Con el dedo pulgar mojado se alisaba el borde, dándole su forma característica en bisel; finalmente, se presionaba con el puño cerrado para obtener el fondo (Kalsbeek, 1980, p. 10).

Otros autores argumentan que los BRB eran producidos a partir del revestimiento con arcilla de agujeros de forma troncocónica excavados en el suelo ex profeso. Las marcas de nudillos y de dedos que aparecen en el interior de los cuencos serían el resultado de este proceso de fabricación. Este método tenía como principal ventaja que el artesano podía predeterminar con precisión la capacidad de cada vaso y reproducir grandes cantidades del mismo tamaño y con poco esfuerzo una vez que los moldes estuvieran preparados (Johnson, 1973, pp. 130-131; Beale, 1978, p. 299). Se ha sugerido, incluso, la posible existencia de conjuntos o alineaciones de moldes excavados en el suelo para facilitar la producción masiva (Strommenger, 1980, p. 58). Esta hipótesis fue testada de forma experimental y dio como resultado, según el autor del ensayo, BRB muy similares a los del período de Uruk elaborados en aproximadamente 60 segundos (Johnson 1973, p. 131).

Por último, se ha propuesto que los BRB fueron fabricados a partir de un molde que podía ser de cerámica o, también, de madera o arcilla cruda (Balfet, 1980, p. 78). No se tiene constancia, sin embargo, del hallazgo de este tipo de moldes en el registro arqueológico urukeo. De ser cierta esta tesis, cabrían dos explicaciones: bien no se han conservado (si eran de materiales orgánicos o frágiles), bien este tipo de piezas no han sido identificadas correctamente entre la cultura material urukea. Es posible que estos moldes portátiles no fueran diferentes a los BRB propiamente dichos y se hayan interpretado, por error, como cuencos (Jamieson, 1989-1990, p. 16).

\subsection{Estudio morfológico}

Las principales características morfológicas del BRB son su perfil troncocónico, habitualmente asimétrico o torcido, así como su borde en bisel (fig. 4). Las dimensiones medias de los ejemplares estudiados son las siguientes:

- Diámetro de la boca: $17 \mathrm{~cm}$.

- Diámetro de la base: $7 \mathrm{~cm}$.

- Altura del vaso: $8 \mathrm{~cm}$.

- Grosor de la pared: $1.3 \mathrm{~cm}$.

- Bisel: $45^{\circ}$.

El simple análisis ocular de la superficie, tanto exterior como exterior, de los BRB completos hallados en Tell Humeida (Siria) y de los procedentes de Tell Qannas (Siria), Tello (Iraq) y Susa (Irán), conservados hoy en Bruselas y París, ha servido para documentar toda una serie de detalles precisos sobre la posible técnica de fabricación.

La superficie exterior se caracteriza por ser tosca, irregular, rugosa y estar agrietada o craquelada, debido con toda probabilidad a un tratamiento de desecación intencionado (fig. 5). No hay huellas de manipulación con las manos. Finalmente, se observa que la base ha sufrido una torsión, que es consecuencia de un movimiento circular (fig. 6). 
SPAL 30.2 (2021)

\section{3-123}

ISSN: 1133-4525

ISSN-e: 2255-3924

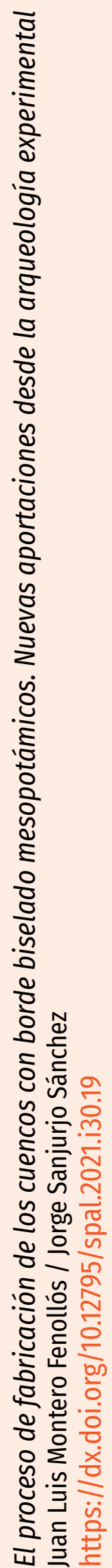

Figura 4. Ejemplar de BRB hallado en Tell Humeida, Siria (Foto: Eloy Taboada-PAMES).

Figura 5. Superficie exterior craquelada de un BRB de Susa, Irán, Sb.18774 (Foto: Juan Luis Montero-PAMES).

Figura 6. Detalle de la torsión sufrida en la base de un BRB de Tell Qannas, Siria, 0.4123 (Foto: Juan Luis Montero-PAMES).
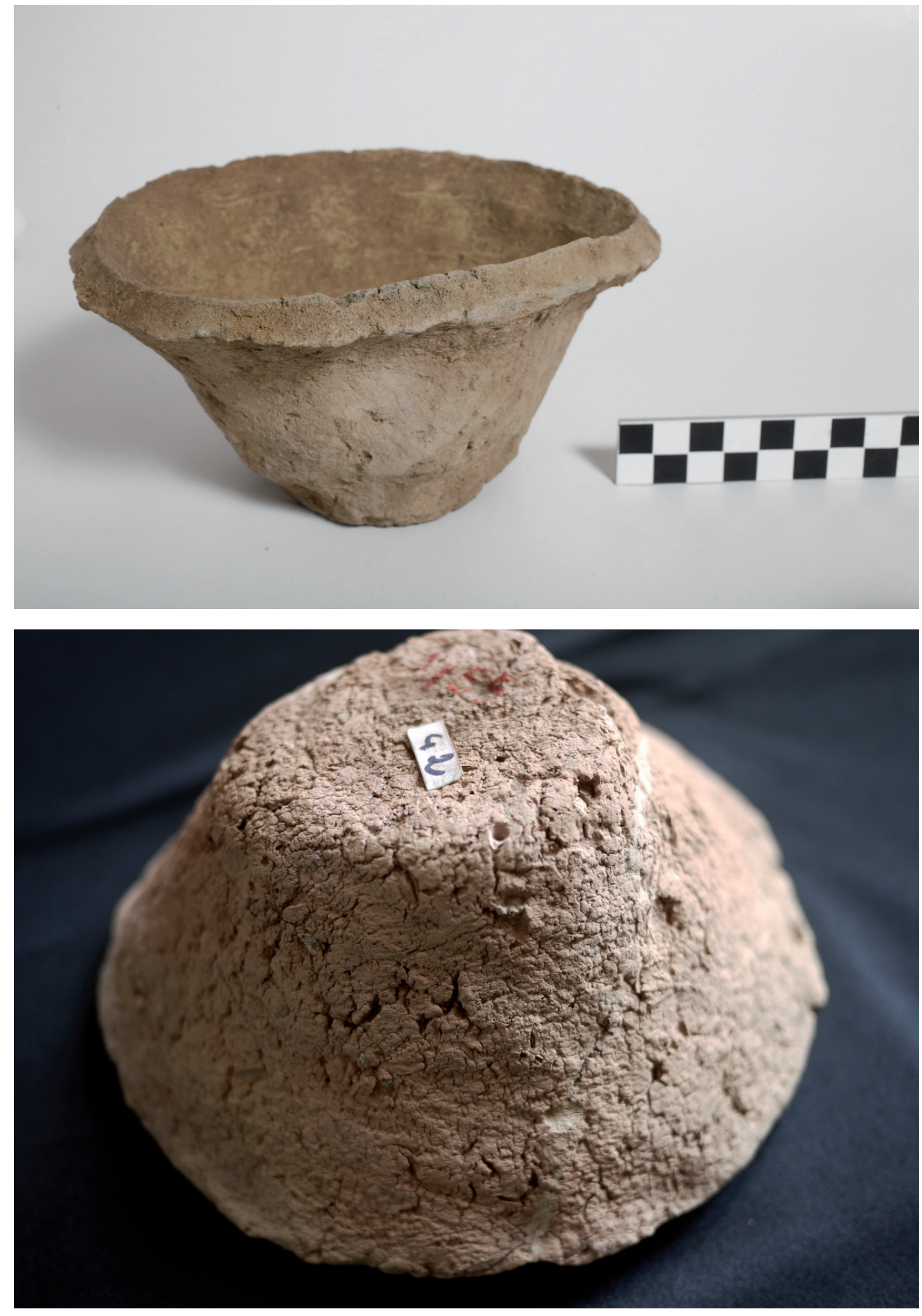
SPAL 30.2 (2021)

\section{$103-123$}

ISSN: $1133-4525$ ISSN-e: 2255-3924
Las características de la superficie interior son totalmente distintas. Presenta un aspecto alisado para el que fue necesario el empleo de agua y se identifican con claridad la presencia de anillos concéntricos (fig. 7). Esto nos indica la existencia de un movimiento rotativo. El fondo presenta huellas impresas de dedos y de nudillos originadas por la presión efectuada con la mano para darle forma al cuenco (fig. 8).

Por último, la inspección visual de los BRB fragmentados de Tell Humeida sirvió para observar la presencia de abundante desgrasante vegetal, que en su origen sería esencialmente paja triturada (fig. 9).

Figura 7. Detalle de los anillos concéntricos del interior de un BRB de Susa, Irán, Sb.18774 (Foto: Juan Luis Montero-PAMES).

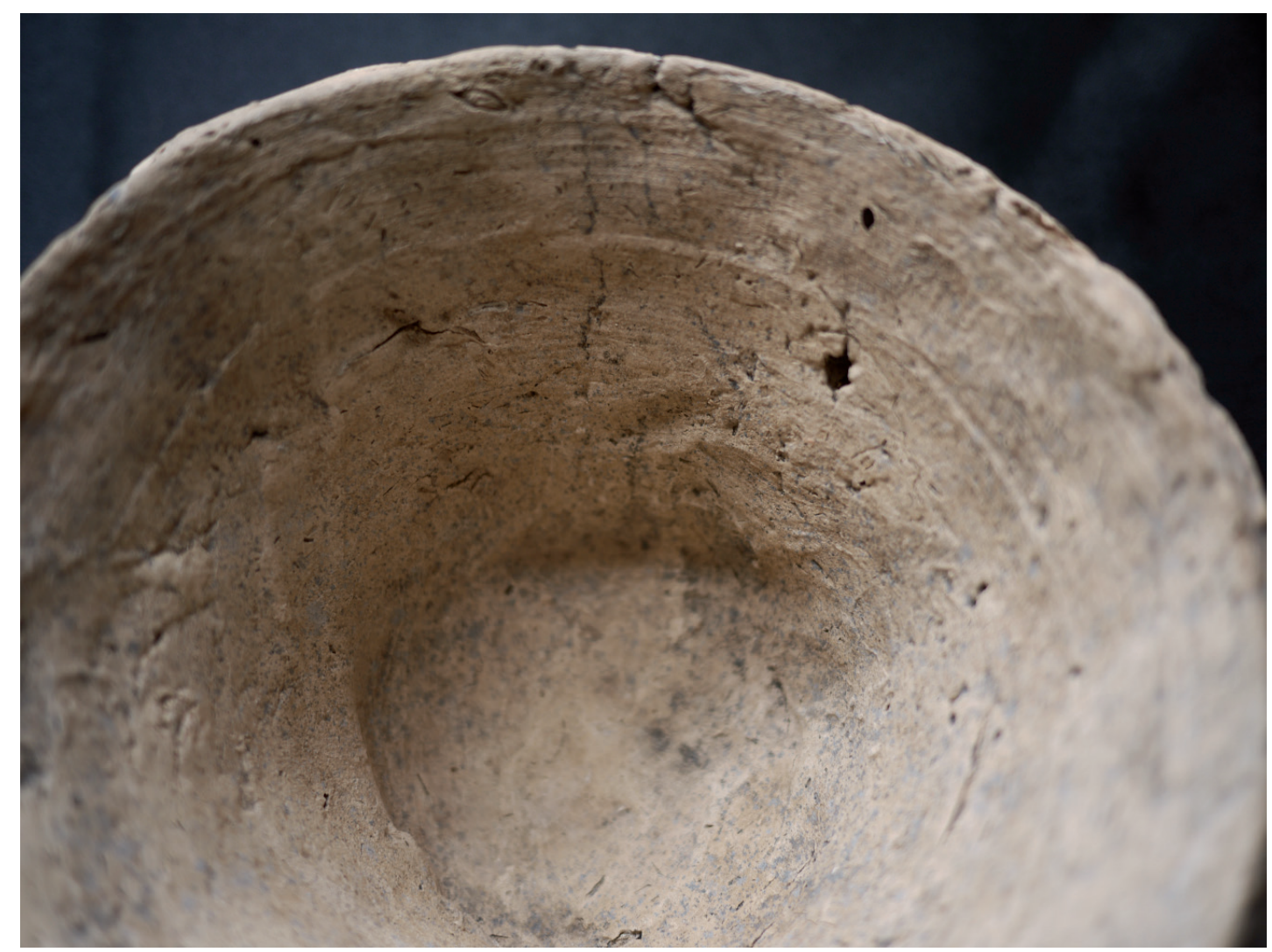

Figura 8. Marcas de dedos en el interior de un BRB de Tell Qannas, Siria, 0.4077 (Foto: Juan Luis Montero-PAMES).

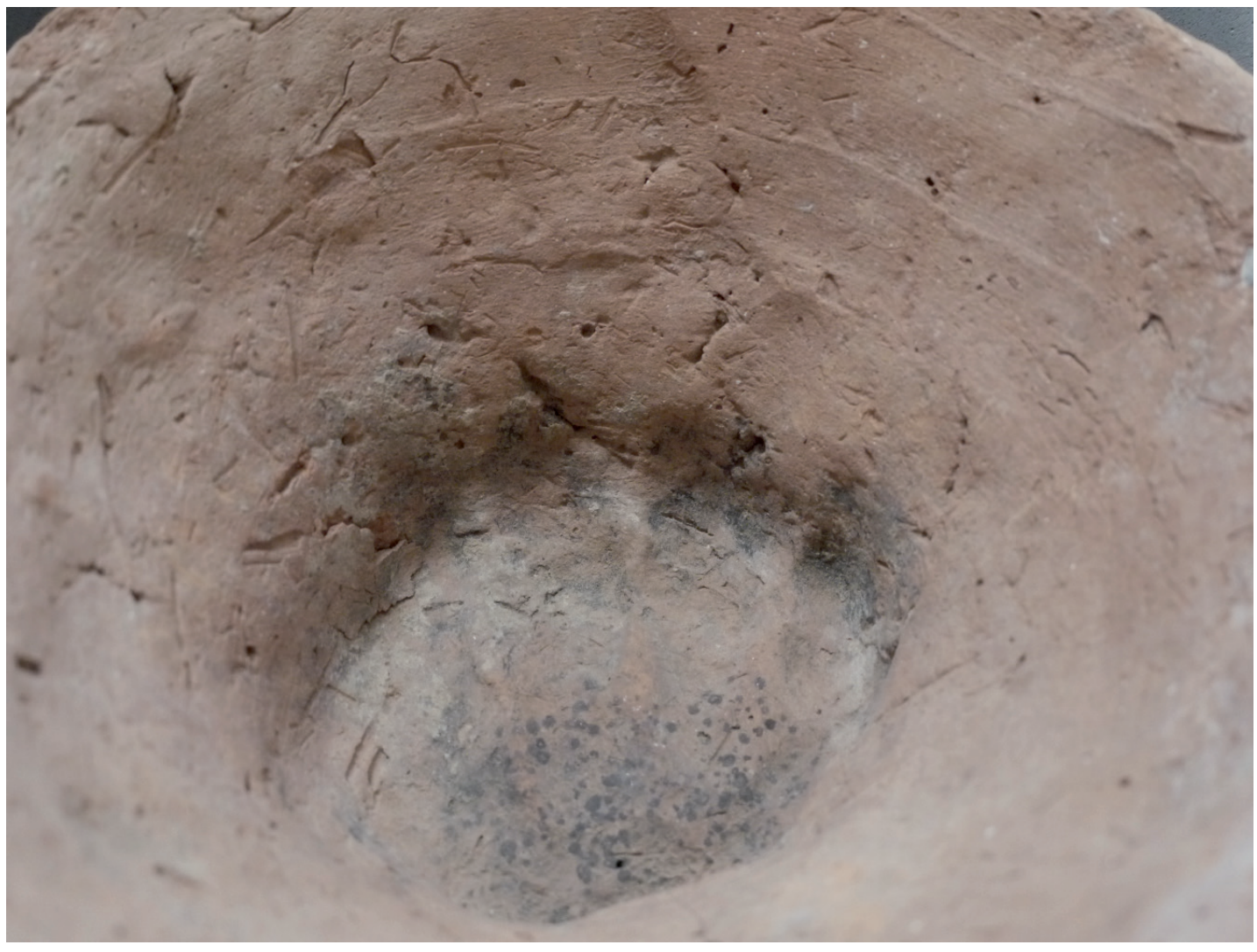


SPAL 30.2

$(2021)$

\section{$103-123$}

ISSN: 1133-4525 ISSN-e: 2255-3924
Figura 9. Sección de un fragmento de BRB de Tell Humeida, Siria (Foto: Eloy Taboada-PAMES).

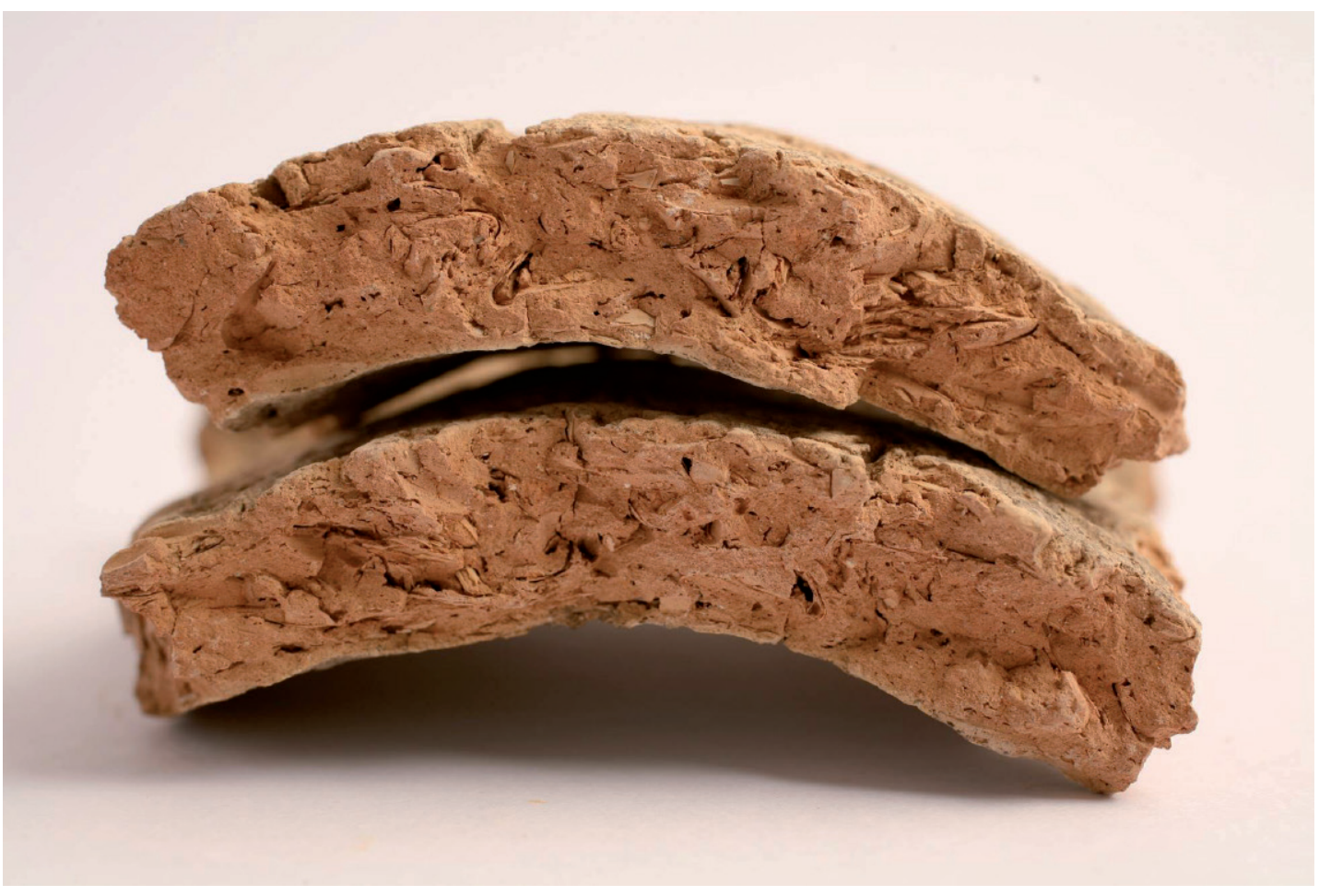

\subsection{La composición geoquímica}

Existen pocos estudios acerca de la composición elemental y mineral de los BRB. Los escasos estudios analíticos existentes han sido realizados en ejemplares procedentes de yacimientos del valle del Éufrates, en Siria, y del sur de Iraq. Algunos de estos estudios han analizado su composición en elementos mayoritarios y minoritarios por fluorescencia de rayos- $X$, comparando muestras de un solo yacimiento (Stephen y Peltenburg, 2002).

En nuestra investigación hemos comparado yacimientos del Medio Éufrates sirio combinando elementos mayoritarios, minoritarios y traza por medio de fluorescencia de rayos- $X$, análisis por activación de neutrones y espectrometría de masas con plasma acoplado inductivamente (Sanjurjo Sánchez et al., 2016). Este estudio permitió analizar muestras de dos yacimientos situados en una distancia máxima aproximada de $180 \mathrm{~km}$ a lo largo del valle: Tell Humeida y Tell Ramadi.

Los resultados obtenidos indicaron una enorme similitud en cuanto a la composición elemental de todas las muestras, independientemente del yacimiento considerado. Esto puede ser interpretado de dos formas:

1. Existía un único centro de producción de BRB para la zona del Éufrates Medio sirio, desde donde luego estos eran redistribuidos;

2. La composición de las arcillas usadas para producir los BRB era muy similar en todo el Éufrates Medio, por lo que la producción podría ser local.

La producción de los BRB y su control no es una cuestión simple, pues puede tratarse de cuencos traídos por gentes emigradas desde el sur de Mesopotamia o importados por población local que deseaba asociarse a lo que representaban culturalmente estos cuencos. También es posible que emigrantes del sur hubieran usado materia prima de la zona para fabricar los BRB o que poblaciones indigenas utilizaran materiales locales para copiar estos vasos foráneos. Los resultados existentes en la actualidad parecen indicar esto último, aunque no es posible aún saber si la producción es local o regional.

Con la finalidad de obtener mayor información que ayude a conocer si la producción de los BRB era local, regional o, incluso, suprarregional, se está ampliando en la 
SPAL 30.2

$(2021)$

\section{$103-123$}

ISSN: $1133-4525$ ISSN-e: 2255-3924

actualidad el número de muestras de nuestro estudio geoquímico a otros yacimientos del Éufrates Medio y de otras zonas de Mesopotamia e Irán.

\subsection{La temperatura de cocción}

Entre los diversos estudios centrados en el conocimiento del proceso de cocción de los BRB, cabe destacar el efectuado a partir de muestras del yacimiento de Uruk, correspondientes al período de Uruk Reciente, que implicó el análisis de varios tipos de cerámica, entre los que se encontraban los BRB. Se calcularon temperaturas de cocción de entre 600 y $1100^{\circ} \mathrm{C}$. Esta estimación fue obtenida realizando estudios de re-cocción y comparando la composición geoquímica, la observación de propiedades físicas por medio de láminas delgadas y estudios de porosidad al agua (Daszkiewicz et al., 2012).

En el marco de nuestro proyecto, se realizaron dos estudios específicos para conocer la temperatura de cocción de los BRB en la región del Medio Éufrates sirio. En el primero de ellos (Sanjurjo Sánchez et al., 2018b), se estimó con gran precisión la temperatura de cocción de cinco muestras de Tell Humeida y tres de Tell Ramadi, utilizando difracción de rayos-X (DRX), espectroscopia infrarroja transformada de Fourier (FTIR), junto con análisis térmico (ATD-ATG) y termoluminiscencia (TL). Las temperaturas estimadas eran muy similares y oscilaban entre los 400 y los $550^{\circ} \mathrm{C}$. En un estudio posterior (Sanjurjo Sánchez et al., 2018c) se realizó un análisis de menor precisión, pero mayor alcance. Este incluía el uso de DRX, FTIR y ATD-ATG y se realizó en más de 60 muestras de BRB de los mismos yacimientos. A estos se sumaron Yerablus Takhtani, Tell Kosak Shamali, Tell Seikh Hassan, Habuba Kabira, Qraya en el Éufrates sirio, y Qars Shemamok, en el valle del Tigris. Las temperaturas obtenidas para la mayor parte de muestras oscilaban entre los $500^{\circ} \mathrm{C}$ y los $650^{\circ} \mathrm{C}$. No se observaron diferencias reseñables entre las temperaturas obtenidas para las muestras de los diferentes yacimientos. Solo unas pocas superaban esta temperatura, pero siempre eran inferiores a los $900^{\circ} \mathrm{C}$. Estos resultados indican con claridad que los artesanos dedicados a la fabricación de BRB utilizaron el mínimo combustible posible en su producción.

En el experimento realizado por nuestro equipo, las temperaturas de cocción utilizadas fueron de $500-510^{\circ} \mathrm{C}$ en un horno de gas, para intentar reproducir el proceso lo más fidedignamente posible.

\subsection{Una nueva visión desde la arqueología experimental}

Tomando como punto de partida el repertorio de datos obtenido previamente, y con la finalidad de comprobar de forma práctica la validez de las principales hipótesis explicativas sobre el proceso de fabricación de los BRB, se llevó a cabo un experimento en el taller de cerámica Terralume de A Coruña. El principal objetivo era comparar las características tecnológicas de los cuencos elaborados en nuestra investigación con los BRB procedentes de las excavaciones arqueológicas.

En primer lugar, se preparó, de acuerdo con la información geoquímica lograda en el laboratorio, una pasta de características similares a la usada en la fabricación de los BRB antiguos a la que se añadió un porcentaje (c $10 \%$ ) de paja triturada.

El primer ensayo consistió en elaborar un BRB mediante el modelado de un cono de arcilla con la única ayuda de las manos (fig. 10). En segundo lugar, se trabajó con un 
SPAL 30.2 (2021)

\section{3-123}

ISSN: 1133-4525 ISSN-e: 2255-3924

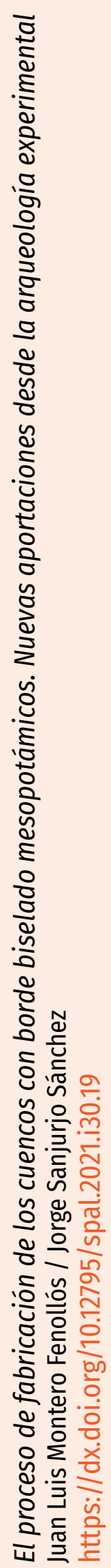

Figura 10. Elaboración de un BRB a mano (Foto: Eloy Taboada-PAMES).
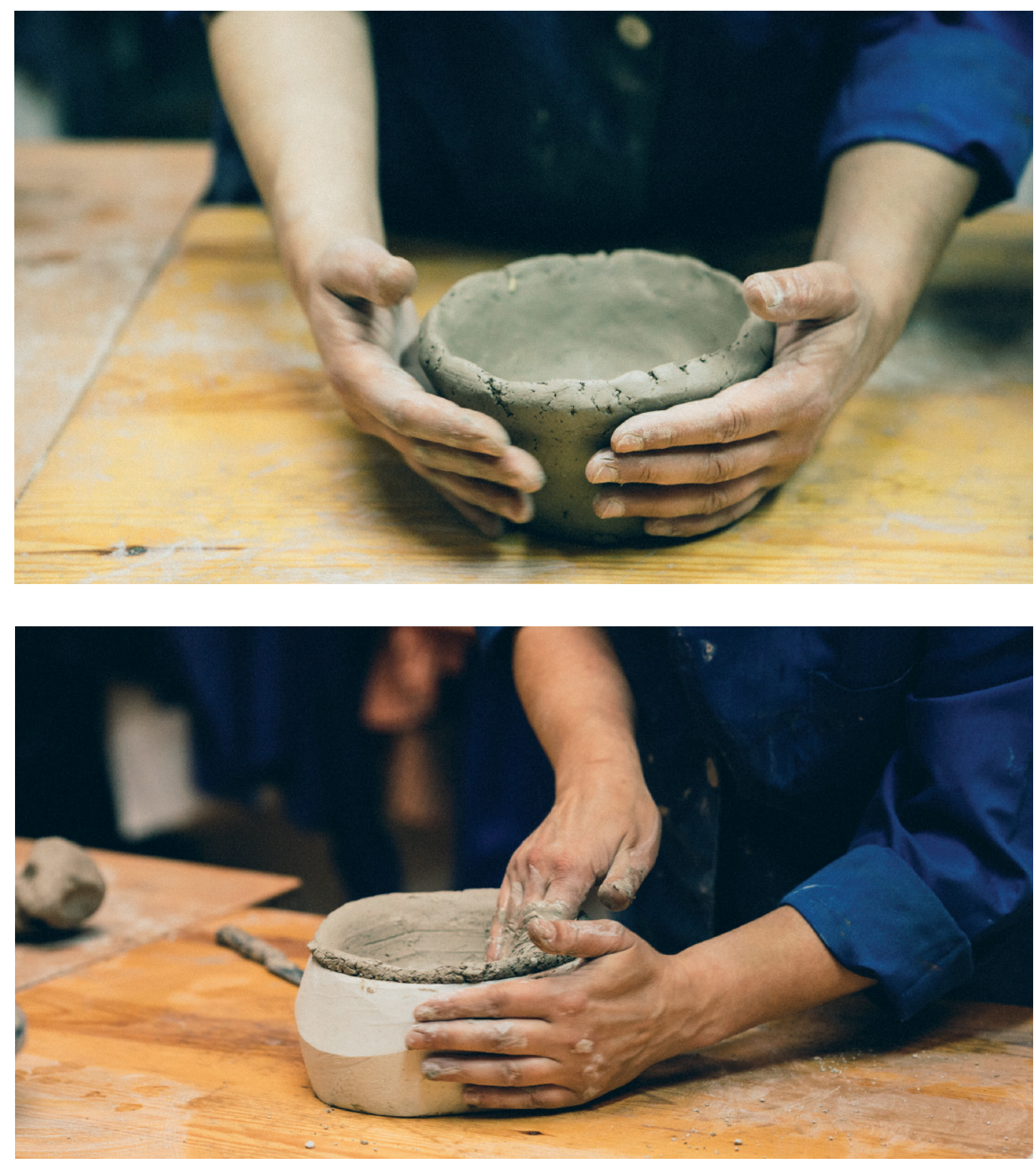

Figura 11a y 11b. Moldes de yeso y de cerámica elaborados para la fabricación de BRB (Fotos: Eloy Taboada-PAMES).

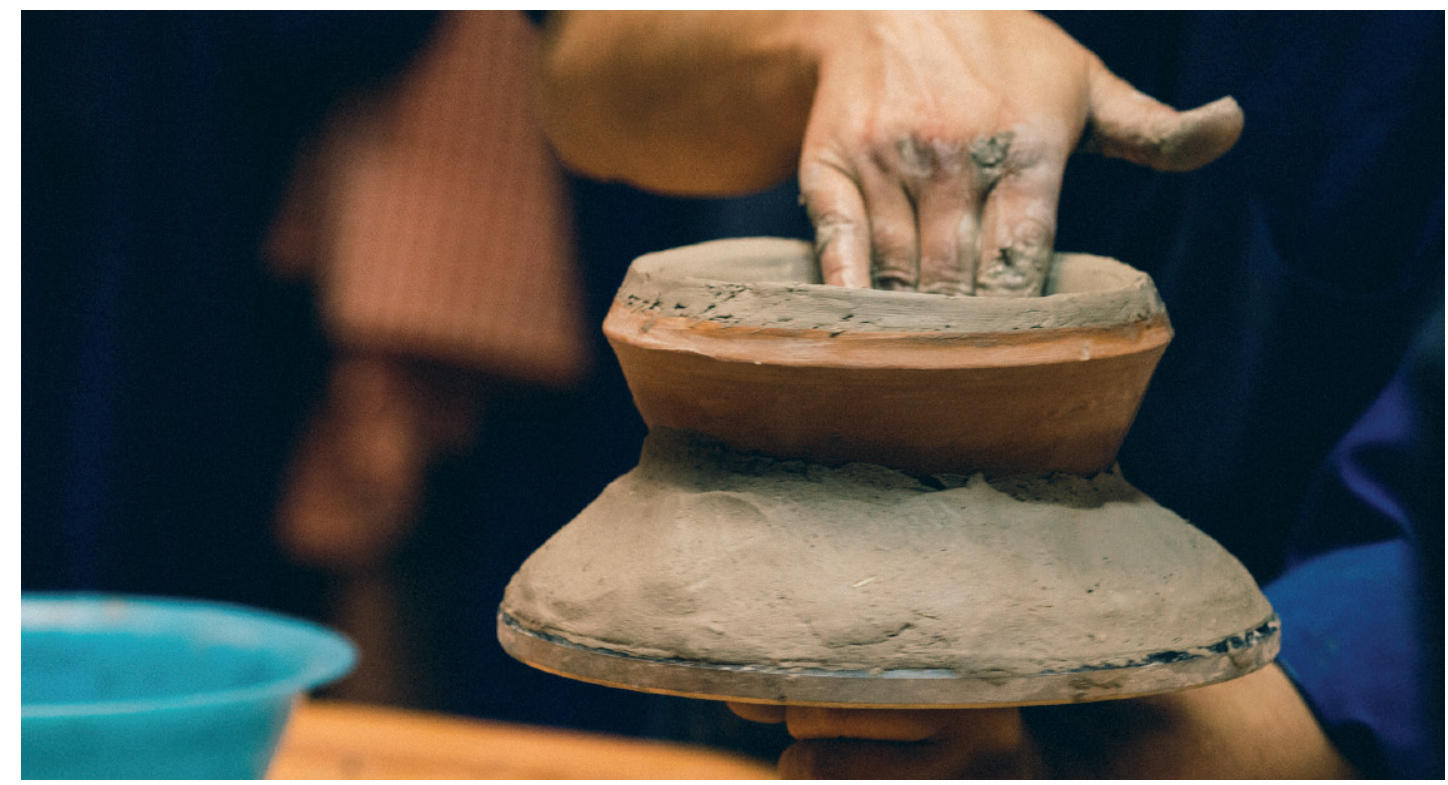


SPAL 30.2 (2021)

\section{$103-123$}

ISSN: $1133-4525$ ISSN-e: 2255-3924 moldes, se fabricaron un total de quince BRB. El tiempo empleado, teniendo en cuenta nuestra inexperiencia, fue inferior a los 2 minutos para cada uno de los ejemplares.

La forma definitiva del cuenco con borde biselado se obtuvo siguiendo los siguientes pasos:

- Introducción de la bola de arcilla rebozada en el molde.

- Movimiento giratorio del molde y presión con un puño cerrado sobre la bola para conseguir la forma.

- Presión con los nudillos para obtener la base.

- Una vez obtenido el grosor deseado de las paredes del cuenco, se alisó la superficie interior con la mano mojada.

- Elaboración del bisel, eliminando la arcilla sobrante con un instrumento afilado.

- Extracción inmediata, tras su elaboración, del cuenco poniendo boca abajo el molde.

- Secado al aire del cuenco con la pasta aún fresca.

- Cocción en un horno moderno de ceramista a una temperatura de $500^{\circ} \mathrm{C}$.

Finalmente, en el tercer experimento se fabricó, a partir de una bola de arcilla recubierta de barro en polvo seco, un BRB en un molde excavado y preparado en un suelo compacto (fig. 13).

Figura 12. Bola de arcilla rebozada en barro seco triturado (Foto: Eloy Taboada-PAMES).

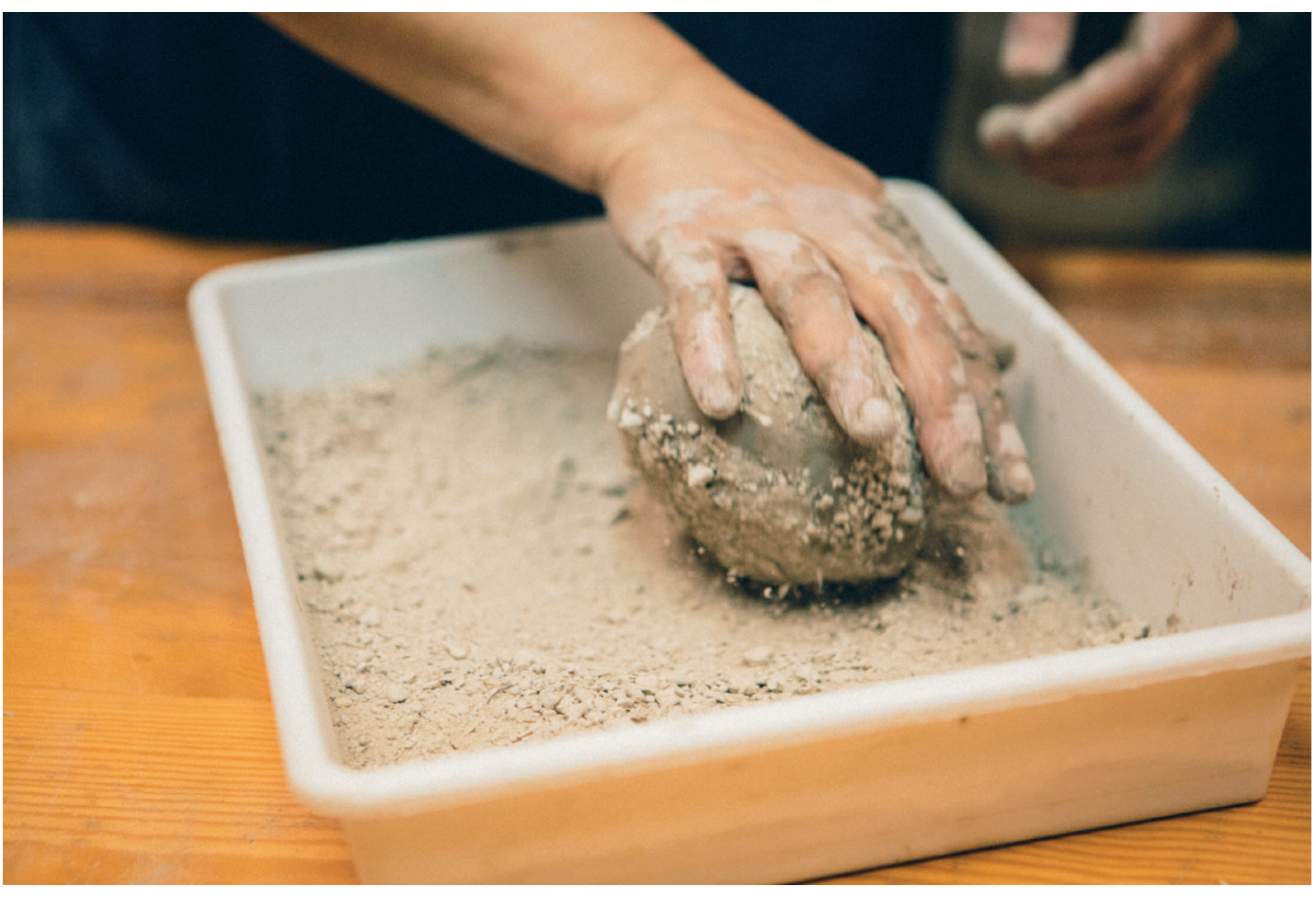

Figura 13. BRB fabricado a partir de un molde excavado en el suelo (Foto: Eloy Taboada-PAMES). 
SPAL 30.2

(2021)

\section{$103-123$}

ISSN: $1133-4525$ ISSN-e: 2255-3924

\section{ANÁLISIS Y DISCUSIÓN DE LOS RESULTADOS}

El ejercicio de arqueología experimental sirvió para realizar una serie de constataciones objetivas, encaminadas a la reconstrucción del proceso tecnológico de fabricación de los BRB durante el período de Uruk Medio y Reciente.

En el caso del cuenco hecho según la hipótesis que defiende el uso exclusivo de las manos, se observó que tanto la superficie interior como la exterior poseían un aspecto muy similar, que era debido a la manipulación y transformación, solo con los dedos, del cono de arcilla con el que se inició la elaboración. Sin embargo, sabemos que el acabado de las dos superficies de los BRB urukeos era totalmente diferente. Así mismo, se constató la complicación de obtener un borde en bisel regular y, sobre todo, la imposibilidad de conseguir dos vasos iguales o comparables.

En cuanto a la forma inicial de la materia prima utilizada por el alfarero para producir los BRB en moldes, se verificó que el uso de tortas circulares provocaba la aparición de pliegues o arrugas en la superficie exterior de los cuencos, que eran originados por la adaptación de la torta plana a la forma troncocónica del molde (fig. 14). Por el contrario, el uso de bolas de arcilla corregía este problema técnico. Así mismo, se comprobó que el rebozado con arcilla seca triturada contribuía a desecar la superficie exterior. Esta técnica facilitaba el desmoldeado de la pieza recién fabricada. El uso de este polvo seco era, además, el responsable del aspecto rugoso y craquelado que presentaban por fuera los cuencos originales.

En el BRB fabricado en un molde fijo excavado en el suelo se atestiguaron varios problemas, que enumeramos a continuación. El primero de ellos fue la extracción en vertical del cuenco aún fresco y su lento proceso de secado en un molde de tierra. El segundo fue la imposibilidad de hacer el característico borde en bisel, pues con este método solo podía ser plano. La tercera dificultad fue poder ejecutar un movimiento de $360^{\circ}$ con la mano en el interior del vaso, como se deduce de los anillos concéntricos que se observan en la superficie interior de los BRB auténticos.

Figura 14. Pliegues en la superficie exterior de BRB elaborados a partir de una torta circular (foto: Juan Luis Montero-PAMES).

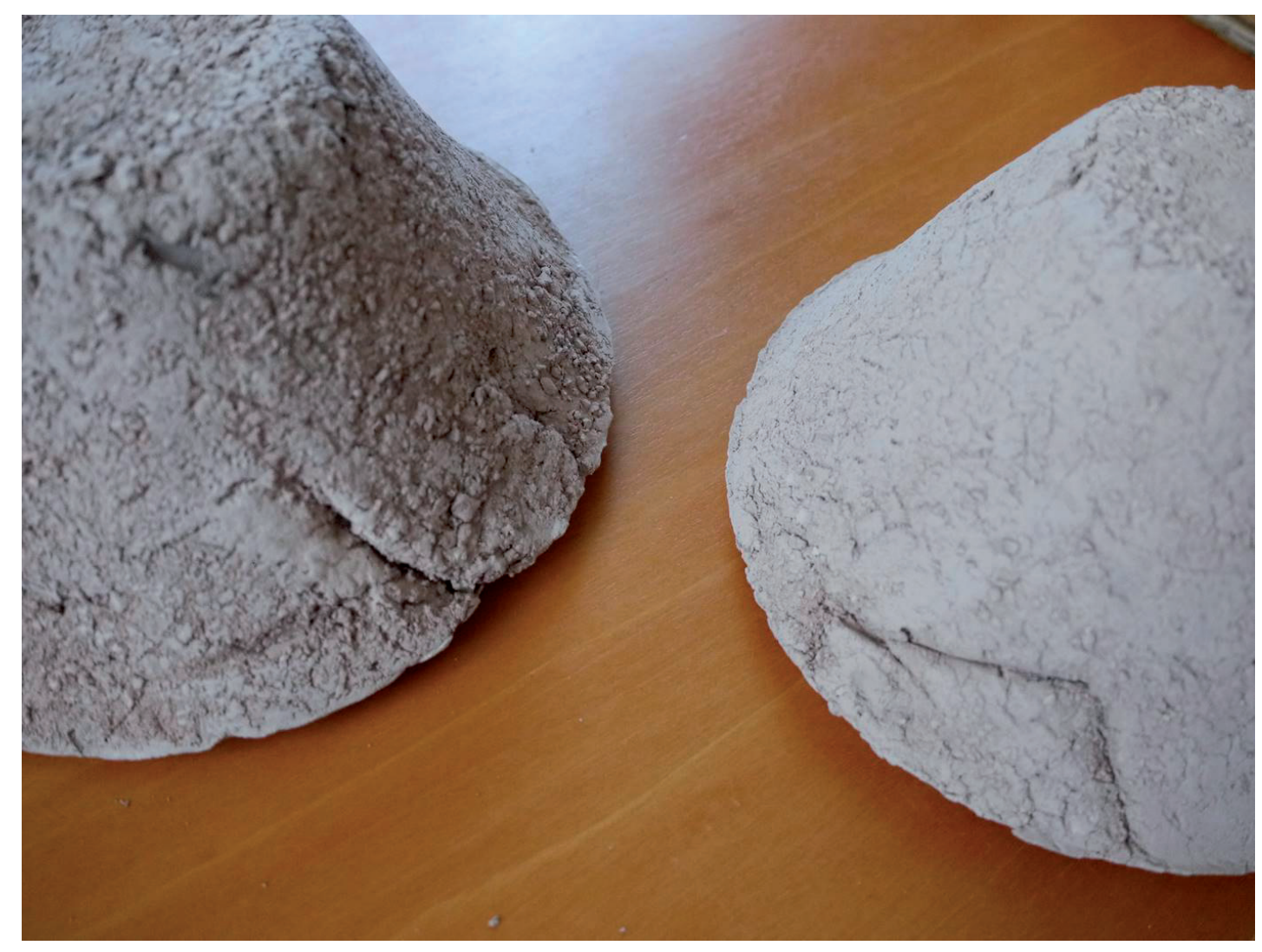


SPAL 30.2 (2021)

\section{$103-123$}

ISSN: $1133-4525$ ISSN-e: 2255-3924
En cuanto a los vasos fabricados con la ayuda de un molde portátil, independientemente del material con el que este estuviera hecho, el resultado fue muy similar a los BRB elaborados en tiempos de la cultura de Uruk (fig. 15): en el aspecto de ambas superficies (agrietado y craquelado en el exterior; y alisado y con anillos concéntricos en el interior); en la fácil elaboración del borde en bisel (mediante la eliminación de la arcilla sobrante del molde con la ayuda de un útil cortante, fig. 16); en las huellas digitales en el fondo interior del vaso (fig. 17); y en la sencilla extracción del vaso recién hecho del molde (fig. 18). A esto hay que añadir un rapidísimo y barato proceso de fabricación.

Los datos obtenidos en el experimento efectuado respaldan claramente el uso de un molde móvil y descartan la fabricación de los BRB tanto en moldes fijos en el suelo como a mano. El problema es que no conocemos a día de hoy la existencia de moldes de estas características en el registro arqueológico urukeo. Sin embargo, en los Musées Royaux d'Art et d'Histoire de Bruselas se conserva, procedente de las excavaciones belgas en Tell Qannas (norte de Siria), un objeto (no de inventario: 0.4071) que por sus características bien podría haber sido un molde empleado para fabricar cuencos con borde biselado (fig. 19). Se trata de un BRB de $17.50 \mathrm{~cm}$ de diámetro revestido con una gruesa capa de adobe de $2.5 \mathrm{~cm}$ de grosor, que fue hallado en una sala de un edificio definido por su descubridor como un templo (Finet, 1982, p. 87; Finet, 1975, p. 154). Otros autores lo interpretan, por el contrario, como un centro de poder (Margueron, 2013, p. 247).

Figura 15. BRB fabricado en el experimento con un molde móvil (Foto: Eloy Taboada-PAMES).

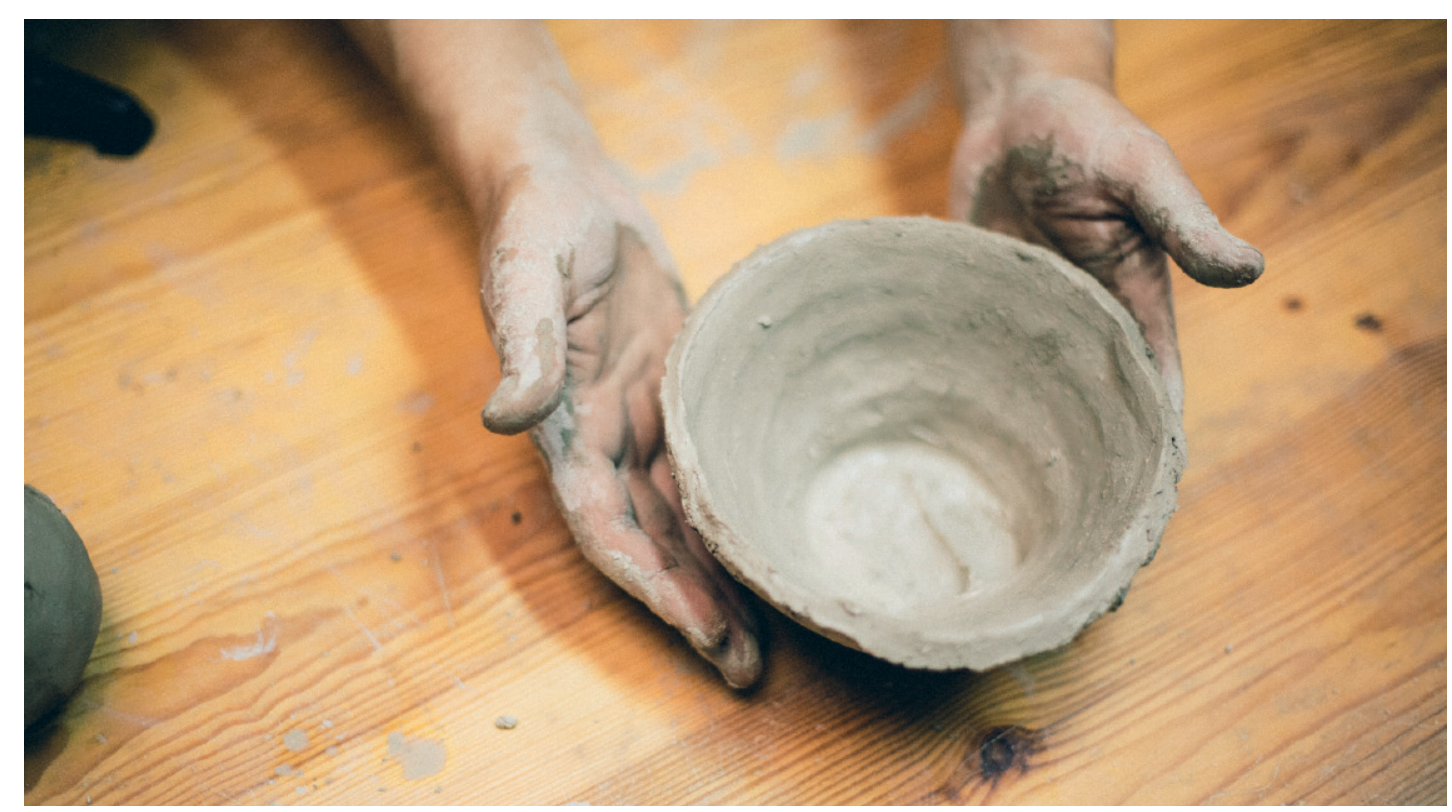

Figura 16. Proceso de obtención del característico borde en bisel de los BRB (Foto: Eloy Taboada-PAMES).

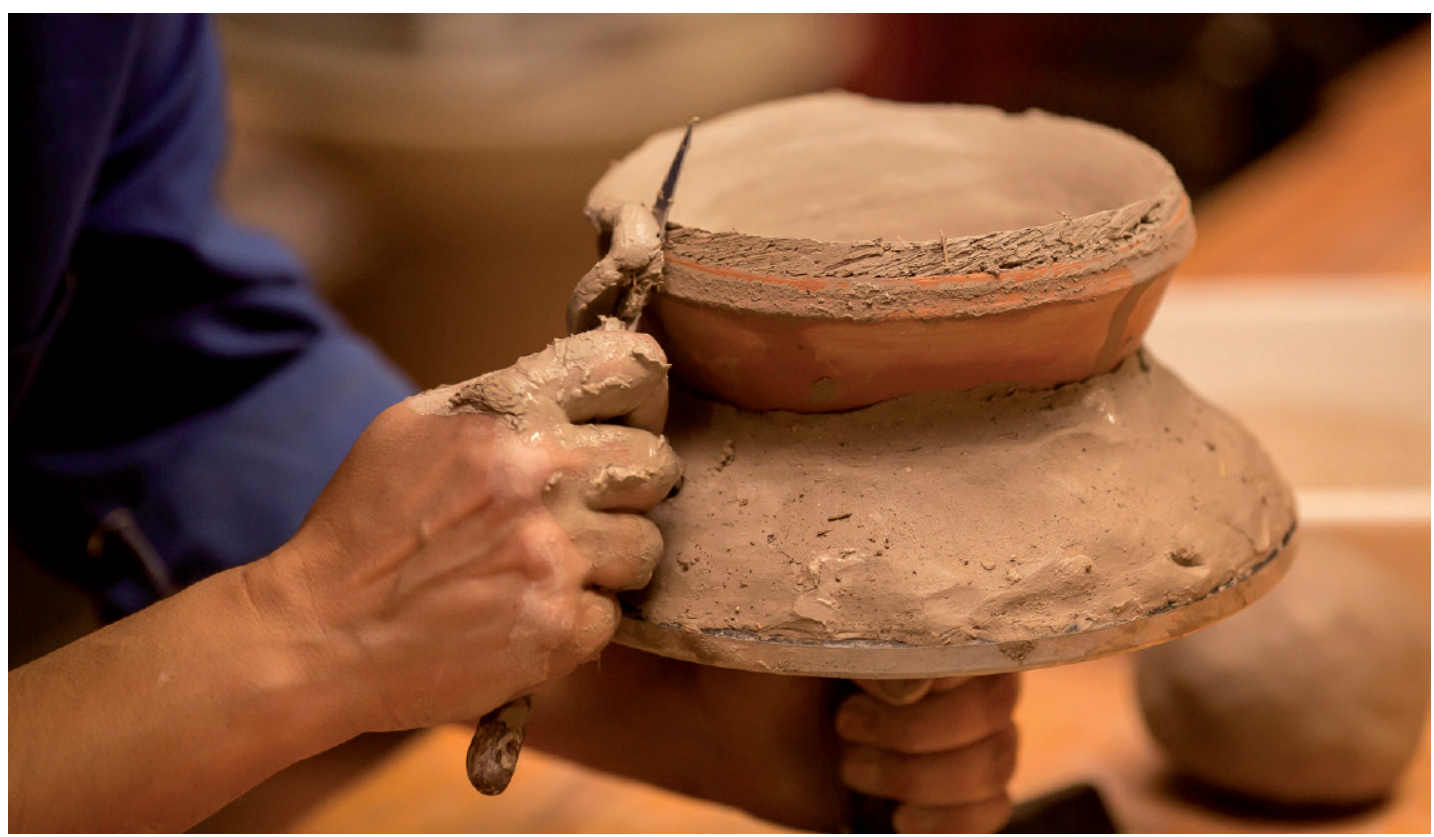




\section{SPAL 30.2 (2021)}

\section{3-123}

ISSN: 1133-4525

ISSN-e: 2255-3924

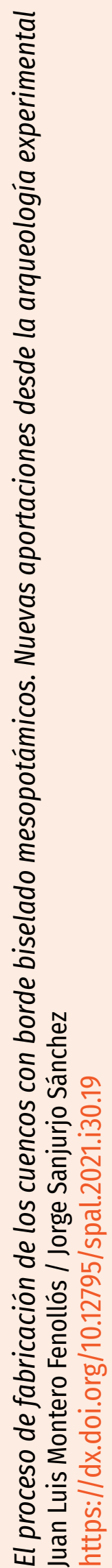

Figura 17. Huellas de dedos en el fondo de un BRB recién elaborado (Foto: Eloy Taboada-PAMES).
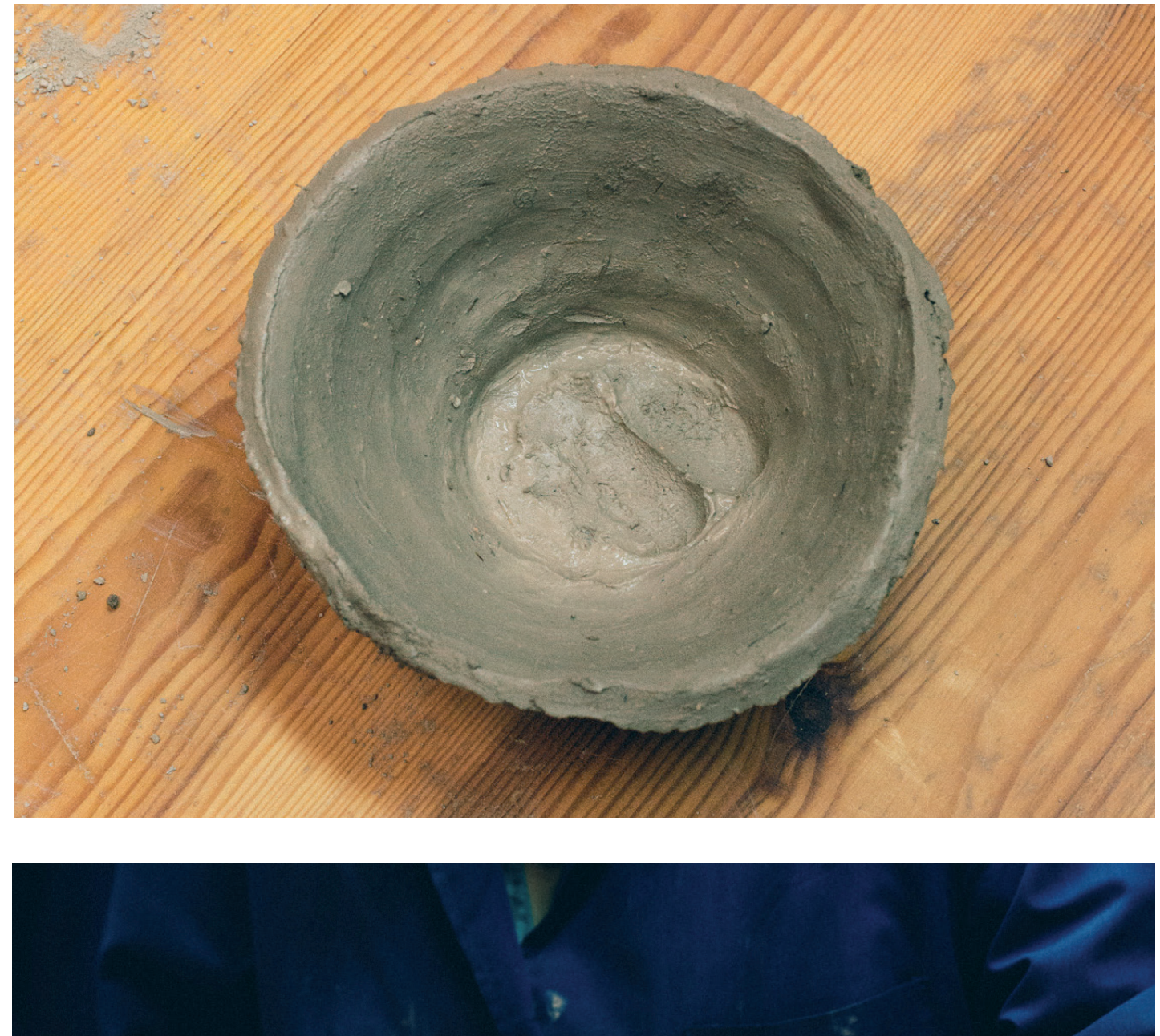

Figura 18. Proceso de desmoldeado de un BRB (Foto: Eloy Taboada-PAMES).

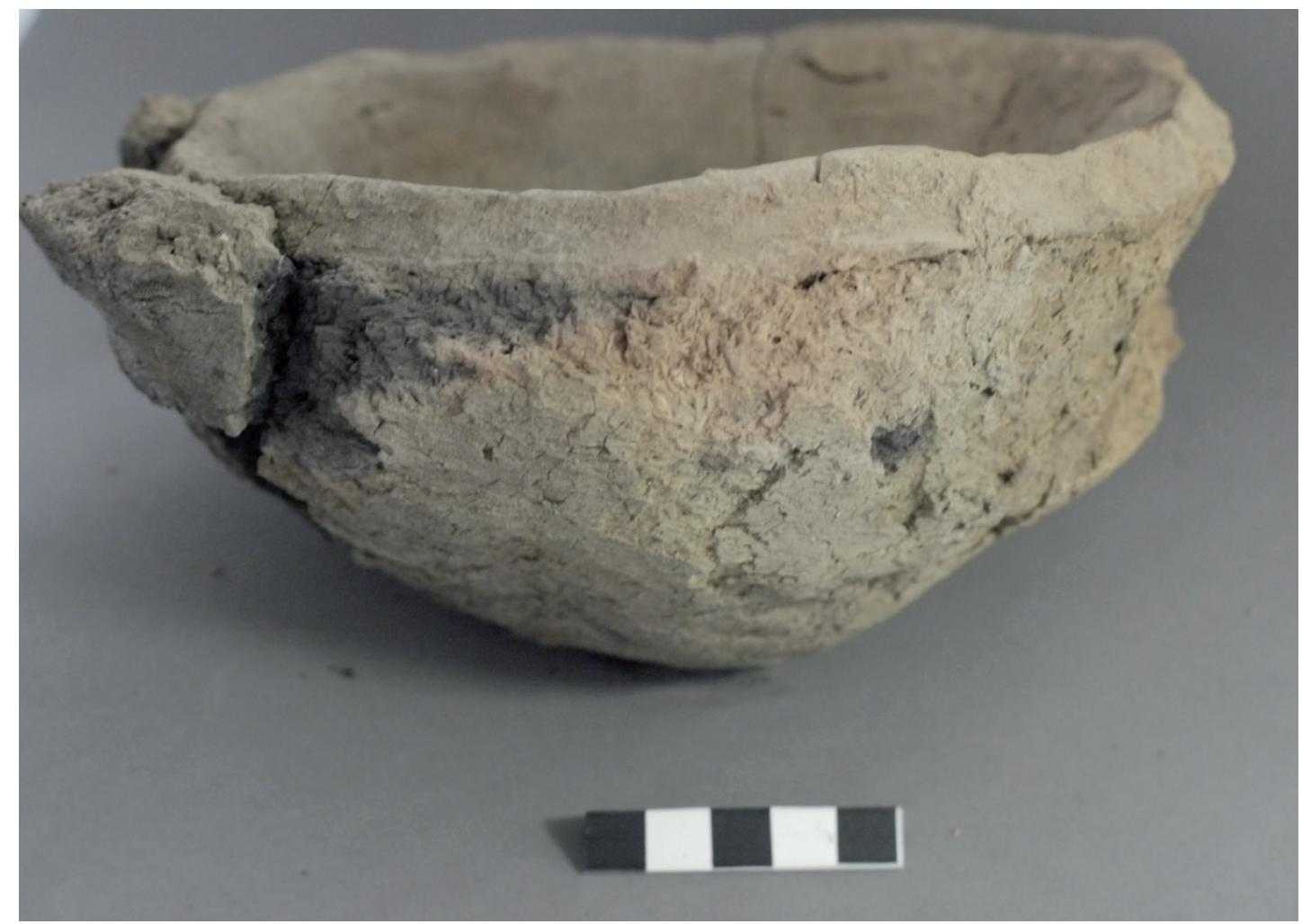

Figura 19. Posible molde para la fabricación de BRB de Tell Qannas (Foto: Juan Luis Montero-PAMES). 
SPAL 30.2

(2021)

\section{$103-123$}

ISSN: $1133-4525$ ISSN-e: 2255-3924
El estudio realizado a partir de láminas delgadas de muestras de BRB de diversos yacimientos del Medio Éufrates sirio muestra siempre una porosidad importante, siendo el tamaño de los poros muy variable (Sanjurjo Sánchez et al., 2018c), lo que se asocia habitualmente con temperaturas de cocción bajas. Así mismo, se aprecia en algunas muestras que los poros están alineados paralelamente a la superficie. Esta observación coincide con la presencia de huellas de torsión, verificada en algunos cuencos originales, y con el uso de un molde. Estos poros se suelen formar al aplicar una fuerza en una sola dirección. Para comprobar esta posibilidad, se ha comparado la porosidad de un BRB original con la de tres elaborados en el experimento: uno producido a mano, otro a partir de una cavidad excavada en el suelo y un tercero a partir de un molde móvil. Para ello, se ha utilizado tomografía computerizada de rayos- $X$, también denominada TC. Esta técnica consiste en obtener una tomografía de la muestra en 3D, de modo que se puede cuantificar el volumen de una cerámica y de su pasta al identificarse los poros abiertos y cerrados, así como su orientación (Kahl y Ramminger, 2012; Karl et al., 2014). Las TC se han obtenido en la Facultad de Odontología de la Universidade de Santiago de Compostela, usando un CBCT Imaging Science International I-CAT 17-19, Modelo number 1-10-3-0, equipado con un emisor de rayos X de 120 kVp y 3-8 mA por Radiación Pulsada (15 pulsaciones por segundo), que cuenta con un Captador de Imagen de Panel de Silicio Amorfo (fabricado por VARIAN) de $20 \mathrm{~cm} \times 25 \mathrm{~cm}$ que permite obtener una Escala de Grises de 14 bits (16384 niveles). El tamaño de Píxel / Vóxel utilizado ha sido de $0.2 \mathrm{~mm}$ y las dimensiones de escaneado de $17 \mathrm{~cm}$ (diámetro) x $13 \mathrm{~cm}$ (altura). El tiempo de exposición usado fue de 10 segundos y la dosis de radiación a la que se expusieron las muestras fue de $32 \mu \mathrm{Sv}$.

Cuando se comparan las TC obtenidas del BRB original y de los experimentales, se observan claras variaciones en la porosidad y en la disposición de poros, pero apenas se identifican diferencias en el desgrasante mineral (fig. 20). La aplicación de una presión unidireccional ocasiona una disposición del desgrasante y de los poros con tendencia a quedar orientados en perpendicular a la dirección de aplicación de la presión (Rye, 1988; Berg, 2008), esto es, en paralelo a la superficie. Esto permite diferenciar el método del moldeado del modelado manual, aunque este último pueda realizarse siguiendo varias técnicas, siendo más o menos sencillo su identificación (Lindahl y Pikirayi, 2010). Tanto en el BRB arqueológico como en los BRB modernos producidos con molde, se observa que la mayor parte de los poros cilíndricos se disponen en una dirección preferente, que es paralela a la de la superficie interna y externa del cuenco. Este hecho se aprecia tanto en posición frontal como sagital (fig. 20: A, B, E, F), lo que indica que la presión se ha aplicado en la cara interna y externa del BRB. Sin embargo, ni en la reproducción realizada en un molde en el suelo (fig. 20: C) ni en el ejemplar hecho simplemente a mano (fig. 20: D) hay una disposición unidireccional de los poros. En ambos casos, la distribución de poros es claramente aleatoria. Esto es debido a que se aplicó una menor presión en las caras interna y externa del cuenco y a que se ejerció presión en otras zonas del vaso durante el proceso de elaboración (Rye, 1988). En el caso del BRB moldeado en el suelo, la técnica de elaboración no permite aplicar la presión en las mismas condiciones e intensidad que en un molde móvil, por lo que la orientación de la paja del desgrasante no es tan evidente. No obstante, a pesar de las evidencias encontradas, al haberse estudiado solo un fragmento de cada tipo (suelo y a mano), esta observación puede considerarse menos concluyente que las anteriores (molde). 


\section{SPAL 30.2 $(2021)$}

\section{$103-123$}

ISSN: $1133-4525$

ISSN-e: 2255-3924
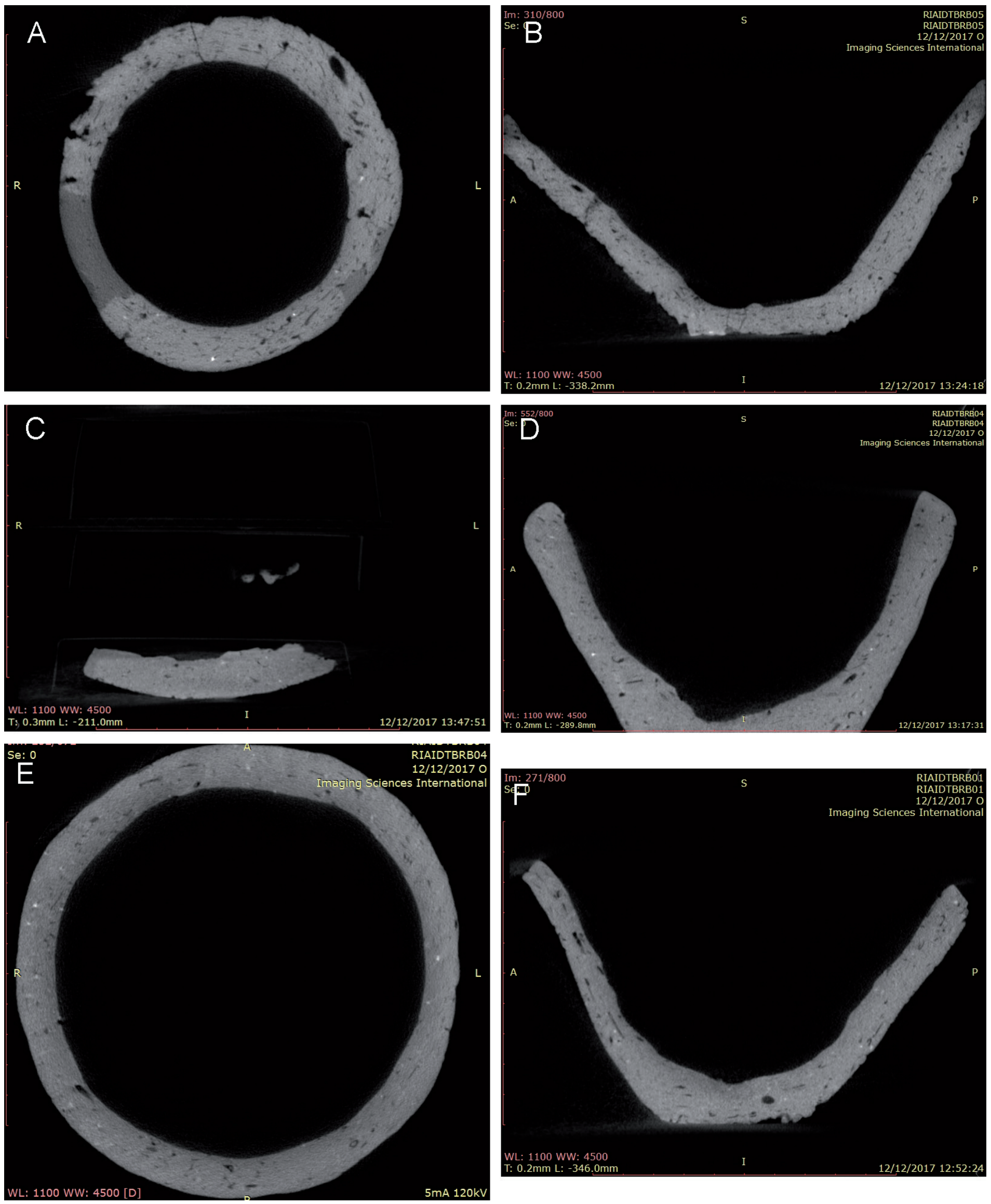

Figura 20. TC de un BRB original restaurado de Tell Humeida en posición sagital (A) y frontal (B) y de BRB producidos experimentalmente: (C) molde en el suelo, (D) modelado a mano, (E) en molde en posición sagital y (F) en molde en posición frontal. 
SPAL 30.2

(2021)

$103-123$

ISSN: 1133-4525

ISSN-e: 2255-3924

Figura 21. Dos BRB elaborados en el experimento (Foto: Juan Luis Montero-PAMES).

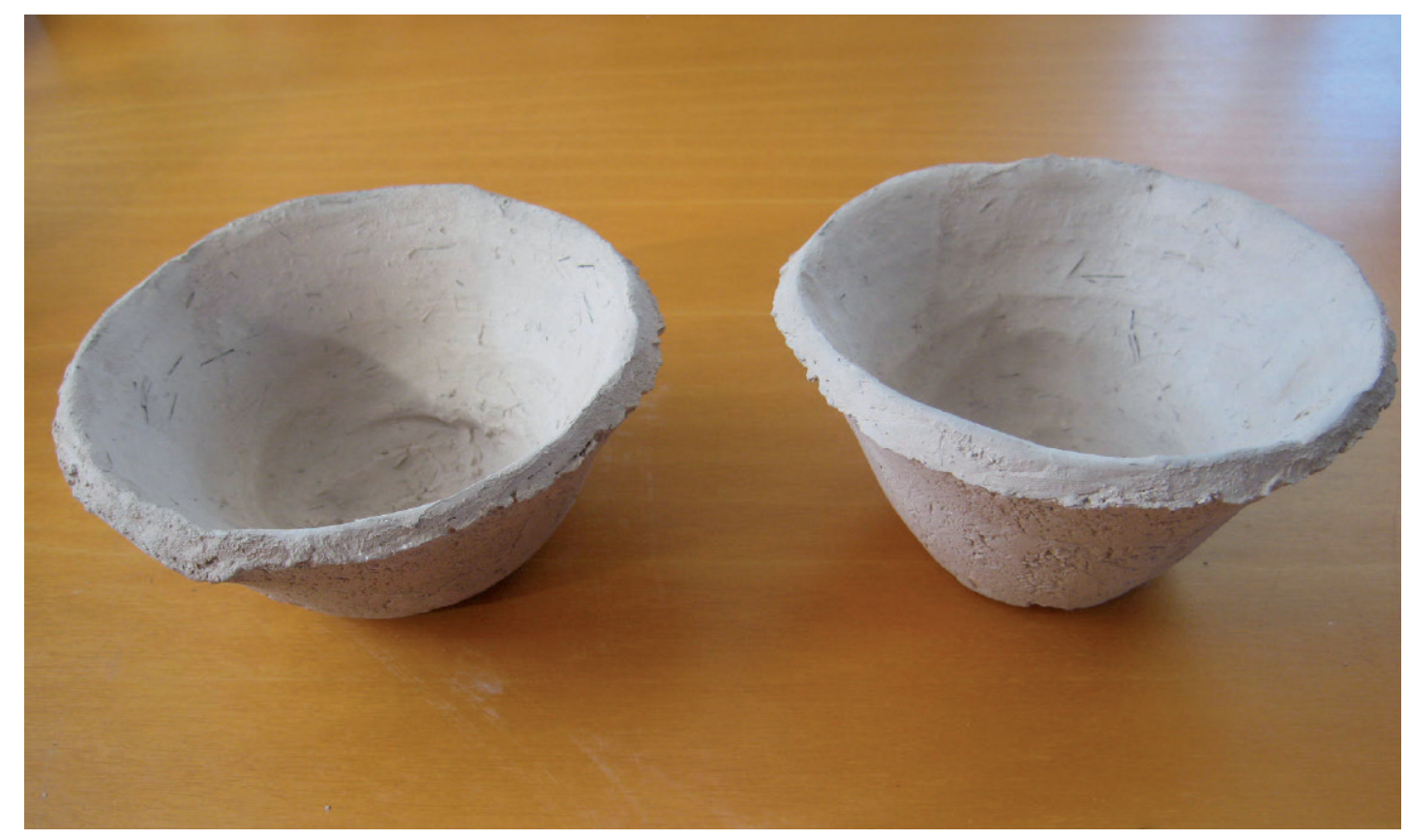

\section{CONCLUSIÓN}

A luz de los datos arqueológicos, de laboratorio y del experimento realizado, podemos reconstruir (fig. 21), por vez primera y con bastantes garantías, cómo era el proceso de fabricación de los BRB durante el período Uruk Medio y Reciente (3600-3000 a.C.):

- No fueron elaborados ni en moldes excavados en el suelo ni exclusivamente con las manos. Por el contrario, los artesanos urukeos se valieron de moldes portátiles o móviles, a pesar de que el uso del torno de alfarero ya era habitual. Con toda probabilidad, los moldes eran BRB (posiblemente los más grandes, de diámetro superior a $17 \mathrm{~cm}$ ) reforzados con barro o adobe por el exterior para facilitar su manejo.

- La materia prima se utilizaba en forma de bola de arcilla y no de torta plana, de un peso de 800-900 g. Una vez cocido el vaso en el horno del experimento, el peso se reduce aproximadamente en $200 \mathrm{~g}$. Uno de los BRB hallados en Tell Humeida pesa hoy $680 \mathrm{~g}$.

- El aspecto exterior (tosco, rugoso, agrietado o craquelado) es debido al uso de arcilla seca triturada para evitar que el cuenco se adhiera al molde y facilitar su extracción recién fabricado. La torsión observada en la base está relacionada con el proceso de relleno del molde y con el movimiento circular empleado en su elaboración.

- El aspecto interior (alisado y con marcas de anillos concéntricos) obedece a un tratamiento final o alisado con agua, que era obtenido haciendo girar el molde sobre sí mismo. La presencia de huellas de dedos en la base tiene su origen en la presión efectuada con los nudillos, bien para adaptar la pasta al molde, bien para corregir ligeramente la asimetría que en ocasiones ocurría al apoyar en el suelo el BRB (con la arcilla aún fresca) tras ser extraído del molde.

- Se trata de un vaso de cerámica de factura poco cuidada y rápida. De hecho, el tiempo empleado en su elaboración, según nuestro experimento, oscilaría entre 1 y 2 minutos. Un experimentado artesano urukeo los fabricaría en un tiempo aún menor.

- La cocción se realizaba en hornos de alfarero a baja temperatura $\left(500-600^{\circ} \mathrm{C}\right)$. Dado que la conductividad de la pasta es muy baja, es posible que la temperatura del horno utilizado, sobre todo en algunas zonas del mismo, fuese algo superior. Esto explicaría la variabilidad en las temperaturas estimadas para los cuencos urukeos. 
SPAL 30.2

$(2021)$

\section{$103-123$}

ISSN: $1133-4525$ ISSN-e: 2255-3924
Si finalmente se trata de moldes para pan, como parece probable, los BRB son testimonio material de la vida cotidiana urukea y de la sociedad de la época. No son tecnológicamente sofisticados, pero simbolizan una nueva identidad culinaria y cultural, la que encarna el mundo de Uruk. El pan (con levadura) era un alimento básico que, a mediados del IV milenio a.C., representaba una nueva forma de vida civilizada en el Oriente Próximo antiguo.

\section{AGRADECIMIENTOS}

Los autores desean expresar su gratitud al Département des antiquités orientales del Louvre, a la Section Proche-Orient et Iran de los Musées royaux d'Art et d'Histoire de Bruselas, y a L. Minc, E. Peltenburg, F. Bachmann, D. Sürenhagen, E. Strommenger, Y. Nisiaki y M. G. Masetti-Rouault por permitirnos tanto el acceso a sus colecciones, como por las muestras de BRB urukeos facilitadas para el presente estudio. Este agradecimiento es extensible a Susana González Amado, ceramista responsable del taller Terralume, en A Coruña, por su colaboración en el experimento de reproducción de BRB.

Esta investigación se ha realizado en el marco del proyecto HAR2010-15866 "Investigaciones arqueológicas en el Medio Éufrates. De la cultura de Uruk al reino de Mari: urbanismo y vida urbana en Mesopotamia", del Ministerio de Ciencia e Innovación.

\section{BIBLIOGRAFÍA}

Algaze, G. (2004) El sistema-mundo de Uruk. Barcelona: Bellaterra.

Algaze, G. (2008) La antigua Mesopotamia en los albores de la civilización. Barcelona: Bellaterra.

Balfet, H. (1980) "A propos du métier de l'argile: exemple de dialogue entre ethnologie et archéologie", en Barrelet, M.T. (ed.) L'archéologie de l'Iraq. París: CNRS, pp. 71-82.

Beale, T.W. (1978) "Bevelled-rim bowls and their implications for change and economic organization in the later fourth millennium", Journal of Near Eastern Studies, 37, pp. 289-313.

Berg, I. (2008) "Looking through pots: recent advances in ceramics X-radiography", Journal of Archaeological Science, 35 (5), pp. 1177-1188.

Bernbeck, R. (2009) "Class conflict in ancient Mesopotamia", Anthropology of the Middle East, 4, pp. 33-64.

Butterlin, P. (2018) Architecture et société au Proche-Orient ancien. París: Picard.

Carr, C. (1990) "Advances in ceramic radiography and analysis: Applications and potentials", Journal of Archaeological Science, 17, pp. 13-34.

Chazan, M.y Lehner, M. (1990) "An ancient analogy: pot baked bread in ancient Egypt and Mesopotamia", Paléorient, 16, pp. 21-35.

Crüsermann, N., van Ess, M., Hilgert, M. y Salje, B. (eds.) (2019) Uruk: First City of the Ancient World. Los Angeles: Getty Publications.

Daszkiewicz, M., van Ess, M., y Schneider. G. (2012) "Pottery and clay from Uruk, Southern Iraq. Laboratory analysis of pottery fabrics from the Late Uruk to the Seleucid Period", Zeitschrift für Orient-Archäologie, 5, pp. 90-102.

Faivre, X. (2009) "Rations et notion de capacité standard dans la céramique du Proche-Orient", Cahiers des thèmes transversaux ArScAn, 9, pp. 305-319.

Finet, A. (1975) “Les temples sumériens du Tell Kannâs", Syria, 52, pp. 157-174.

Finet, A. (ed.) (1982) Lorsque la royauté descendit du ciel... Les fouilles belges du Tell Kannâs sur l'Euphrate en Syrie. Mariemont: Musée Royal de Mariemont. 
SPAL 30.2

$(2021)$

\section{$103-123$}

ISSN: $1133-4525$ ISSN-e: 2255-3924
Goulder, J. (2010) "Administrator's bread: an experiment-based re-assessment of the functional and cultural role of the Uruk bevel-rim bowl", Antiquity, 84, pp. 351-362.

Hopkinson, B. (2007) "The bevelled rim bowls of Mesopotamia 4000-3000 B.C. and briquetage as molds for draining and drying salt", en Figuls, A. y Weller, O. (eds.) 1a Trobada Internacional d'Arqueologia envers l'explotació de la sal a la Prehistória i Protohistória, Archaeologia Cardonensis, 1. Cardona: Institut de recerques envers la Cultura, pp. 47-51.

Jamieson, A. S. (1989-1990) "Experiments in the manufacture of ancient Near Eastern pottery", The Artefact, 13, pp. 12-27.

Johnson, G. A. (1973) Local exchange and early state development in Southwestern Iran. Ann Arbor: University of Michigan.

Kahl, W.A Ramminger, B. (2012) "Non-destructive fabric analysis of prehistoric pottery using high-resolution X-ray microtomography: a pilot study on the late Mesolithic to Neolithic site Hamburg-Boberg", Journal of Archaeological Science, 39, pp. 2206-2219. https://doi.org/10.1016/j.jas.2012.02.029

Karl, S., Jungblut D., Mara, H., Wittum, G. y Krömker, S. (2014) "Insights into manufacturing techniques of archaeological pottery: Industrial X-ray computed tomography as a tool in the examination of cultural material", en Martinón-Torres, M. (ed.) Craft and science: International perspectives on archaeological ceramics. Doha: Bloomsbury Qatar Foundation, pp. 253-261. http://dx.doi.org/10.5339/uclq.2014.cas.ch27

Kalsbeek, J. (1980) "La céramique de série du Djebel Aruda (à l'époque d’Uruk)", Akkadica, 20, pp. 1-11.

Le Brun, A. (1980) “Les écuelles grossières. État de la question", en Barrelet, M.T. (ed.) L'archéologie de l'Iraq. París: CNRS, pp. 59-70.

Lindahl, A. y Pikirayi, I. (2010) "Ceramics and change: an overview of pottery production techniques in northern South Africa and eastern Zimbabwe during the first and second millennium AD", Archaeological and Anthropological Sciences, 2, pp. 133-149. https://doi.org/10.1007/ s12520-010-0031-2

Liverani, M. (1998) Uruk. La primera ciudad. Barcelona: Bellaterra.

Margueron, J. C. (2013) Cités invisibles. La naissance de l'urbanisme au Proche-Orient ancien. París: Geuthner.

Márquez, I. (2009) "Pain, bière et la culture d'Uruk. De Gilgamesh au bol à bord biseauté", en Barreyra, D.A y del Olmo Lete, G. (eds.) Reconstructing a Distant Past. Ancient Near Eastern. Essays in Tribute to Jorge R. Silva Castillo. Sabadell: Ausa, pp.133-146.

Montero, J. L. (2011) “Le site urukéen de Tell Humeida au Moyen Euphrate syrien. Premières recherches archéologiques", Res Antiquitatis, 2, pp. 205-216.

Montero, J. L. y Sanjurjo, J. (2016) “Nuevas investigaciones sobre la cerámica de Uruk en el Medio Éufrates sirio: los cuencos con borde biselado o bevelled rim bowls. Resultados preliminares", Aula Orientalis, 34, pp. 293-311.

Pollock, S. (2012) "Politics of food in early Mesopotamia centralized societies", Origini, 34, pp. 153-168.

Porter, A. (2012) Mobile pastoralism and the formation of Near Eastern civilizations. Cambridge: Cambridge University Press.

Rothman, M.S. (ed.) (2001) Uruk Mesopotamia and its neighbors. Santa Fe: School of American Research Press.

Rye, O.S. (1988) Pottery technology. Principles and Reconstruction, Manuals on Archaeology, 4. Washington DC: Taraxacum.

Sanjurjo, J., Montero, J.L., Prudêncio, M.I., Barrientos, V., Marques, R. y Dias M.I. (2016) “Geochemical study of beveled rim bowls from the Middle Syrian Euphrates sites", Journal of Archaeological Science Reports, 7, pp. 808-881. https://doi.org/10.1016/j.jasrep.2016.02.018

Sanjurjo, J., Kaal, J. y Montero, J.L. (2018a) "Organic matter from bevelled rim bowls of the Middle Euphrates: Results from molecular characterization using pyrolysis-GC-MS", Microchemical Journal, 141, pp. 1-6. https://doi.org/10.1016/j.microc.2018.05.001 
SPAL 30.2 (2021)

\section{$103-123$}

ISSN: $1133-4525$

ISSN-e: 2255-3924
Sanjurjo, J., Montero, J.L. y Polymeris, G.S. (2018b) "Technological aspects of Mesopotamiam Uruk pottery: estimating firing temperatures using mineralogical methods, thermal analysis and luminescence techniques", Archaeological and Anthropological Sciences, 10, pp. 849-864. https://doi.org/10.1007/s12520-016-0409-x

Sanjurjo, J., Montero, J.L., Barrientos, V. y Polymeris, G.S. (2018c) “Assessing the firing temperature of Uruk pottery in the Middle Euphrates Valley (Syria): Bevelled rim bowls", Microchemical Journal, 142, pp. 43-53. https://doi.org/10.1016/j.microc.2018.06.009

Stephen, F. y Peltenburg, E. (2002) "Scientific analyses of Uruk ceramics from Jerablus Tahtani and other Middle-Upper Euphtrates sites", en Postgate, J.N. (ed.) Artefacts of Complexity: tracking the Uruk in the Near East. Cambridge: British School of Archaeology in Iraq, pp. 173-190.

Strommenger, E. (1980) Habuba Kabira. Eine Stadt vor 5000 Jahren. Mainz am Rhein: Verlag Philipp von Zabern.

Shneider, G. y Daszkiewicz, M. (2019) “Clay, loam, and ceramics in Mesopotamia”, en N. Crüsemann et al. (eds.) Uruk. First city of the ancient world. Los Angeles: Getty Museum, pp. 232-233.

\section{Contribución Autores}

Los dos autores han participado por igual en todas las tareas de la investigación de este proyecto de arqueología experimental. 